\title{
Cognitive radio for vehicular ad hoc networks (CR-VANETs): approaches and challenges
}

\author{
Kamal Deep Singh ${ }^{*}$, Priyanka Rawat ${ }^{2}$ and Jean-Marie Bonnin ${ }^{1}$
}

\begin{abstract}
With growing interest in using cognitive radio (CR) technology in wireless communication systems for vehicles, it is envisioned that future vehicles will be CR-enabled. This paper discusses CR technologies for vehicular networks aimed at improving vehicular communication efficiency. CR for vehicular networks has the potential of becoming a killer CR application in the future due to a huge consumer market for vehicular communications. This paper surveys novel approaches and discusses research challenges related to the use of cognitive radio technology in vehicular ad hoc networks. We review how CR technologies such as dynamic spectrum access, adaptive software-defined radios, and cooperative communications will enhance vehicular communications and, hence, present the potential of transforming vehicle communication in terms of efficiency and safety. Our work is different from existing works in that we provide recent advances and open research directions on applying cognitive radio in vehicular ad hoc networks (CR-VANETs) focusing on architecture, machine learning, cooperation, reprogrammability, and spectrum management as well as QoE optimization for infotainment applications. A taxonomy of recent advances in cognitive radio for vehicular networks is also provided. In addition, several challenges and requirements have been identified. The research on applying CR in vehicular networks is still in its early stage, and there are not many experimental platforms due to their complex setup and requirements. Some related testbeds and research projects are provided at the end.
\end{abstract}

Keywords: CR-VANETs; CRVs; Dynamic spectrum allocation; IEEE 802.11 p; Intelligent transportation systems; Survey; Vehicular communication; V2I; V2R; V2V

\section{Introduction}

Increasing number of vehicles on the road has brought focus on improving road safety as well as in-vehicle entertainment. In tune with this demand, we are witnessing a rise in development of new applications and services for vehicular environments. Some common examples include applications for collision avoidance, safety and traffic monitoring, multimedia streaming, data collection for smart cities in synergy with wireless sensor networks [1], vehicle-to-vehicle communication (V2V), etc. Consequently, vehicular ad hoc network (VANET) has emerged as a new technology that can support such emerging vehicular applications.

A VANET is defined as a spontaneous ad hoc network formed over vehicles moving on the road. Such a network can be formed between vehicles with V2V communication

\footnotetext{
*Correspondence: kamal.singh@telecom-bretagne.eu

1 Department RSM, Telecom Bretagne, 35510 Cesson Sevigne, France

Full list of author information is available at the end of the article
}

or between vehicles and infrastructure with vehicle-toinfrastructure (V2I) communication. Such VANETs in which vehicles can communicate with each other and also with roadside infrastructure provide a means to improve road safety by enabling a number of potential applications for driver assistance, collision warning, traffic information, and monitoring [2-6]. The availability of various applications will improve road safety and vehicular environment.

Dedicated short-range communication (DSRC) [7-10] is a generic name for short-range, point-to-point communication. It is also the name of the older technology mainly used for vehicle to road communication (e.g., toll gate). The channels reserved worldwide in the $5.9 \mathrm{GHz}$ band for such communications are known as DSRC channels. The IEEE 1609 - Dedicated Short-Range Communication Working Group is developing standards for wireless access in vehicular environments (WAVE) and the communication is based on IEEE 802.11p [11,12], which is an amendment to IEEE 802.11 standard in order to support

\section{照 Springer}

2014 Singh et al: licensee Springer. This is an Open Access article distributed under the terms of the Creative Commons

Attribution License (http://creativecommons.org/licenses/by/2.0), which permits unrestricted use, distribution, and reproduction in any medium, provided the original work is properly cited. 
communication in dynamic vehicular environments. IEEE 802.11p standardizes the communication aspects related to physical (PHY) and media access control (MAC).

Currently, the US Federal Communications Commission (FCC) has allocated $75 \mathrm{MHz}$ and the European Telecommunications Standards Institute (ETSI) allocated $30 \mathrm{MHz}$ of spectrum in $5.9 \mathrm{GHz}$ band for the deployment of Intelligent Transportation Systems (ITS) services $[13,14]$. However, a significant rise in vehicular applications, especially in urban environments, with several vehicles, may lead to overcrowding of the band and thereby resulting in degraded vehicular communication efficiency for safety applications, as pointed out in [15]. Moreover, not only safety applications, but also growing demand and usage of in-car entertainment and information systems comprising bandwidth demanding multimedia applications (e.g., video streaming) will lead to congested vehicular networks and spectrum scarcity for IEEE 802.11p-based vehicular applications [15].

In view of this, using cognitive radio (CR) technology in VANETs will enable more efficient radio spectrum usage and, in turn, improve vehicular communication efficiency. Figure 1 illustrates a V2V scenario with cognitive radio which allows for opportunistic spectrum usage, along with the utilization of DSRC channels. Spectrum utilization measurements over the years have indicated an inefficient spectrum allocation and usage with many unused or underused licensed bands over different space and time, for example, spectrum bands for TV broadcasting, resulting in considerable spectrum wastage [16-20]. Consequently, regulatory agencies, such as FCC [21] in the USA that regulates spectrum allocations, have now opened the licensed bands to unlicensed/secondary user (SU) through the use of CR [22-27] in order to enable a more efficient use of the spectrum bands. CR is an emerging technology to improve spectrum usage and alleviate spectrum scarcity by exploiting underused spectral resources through opportunistic spectrum access.

Radio cognition ( $\mathrm{RC}$ ) together with dynamic spectrum access (DSA) strategies present promising approaches that allow unlicensed/secondary users to opportunistically capture and use the spatio-temporally available licensed spectrum holes as long as licensed/primary user (PU) are not perturbed [25]. Though many applications of CR have been proposed for wireless mesh and in ad hoc and cellular networks, employing CR in vehicular (CRV) environments is a relatively novel research subject and some works in that direction have started to appear recently [28-35].

Vehicular networks and vehicular communication can benefit from opportunistic spectrum usage with CR technology [36]. Cognitive radio for vehicular Ad hoc Networks (CR-VANETs) is a fast-emerging application area of CR technology [28]. CR-enabled vehicles can use additional spectrum opportunities in TV bands according to the quality-of-service (QoS) requirements of the applications. However, the research solutions proposed for general-purpose CR networks cannot be directly applied to CR-VANETs. This is because the unique features of vehicular environment such as high mobility and cooperation opportunities need to be taken into account while designing the spectrum management functions for CRVANETs. Unlike static CR scenarios, in CR-VANETs, multiple cooperating vehicles (during busy hours) can

\section{DSRC Channel}

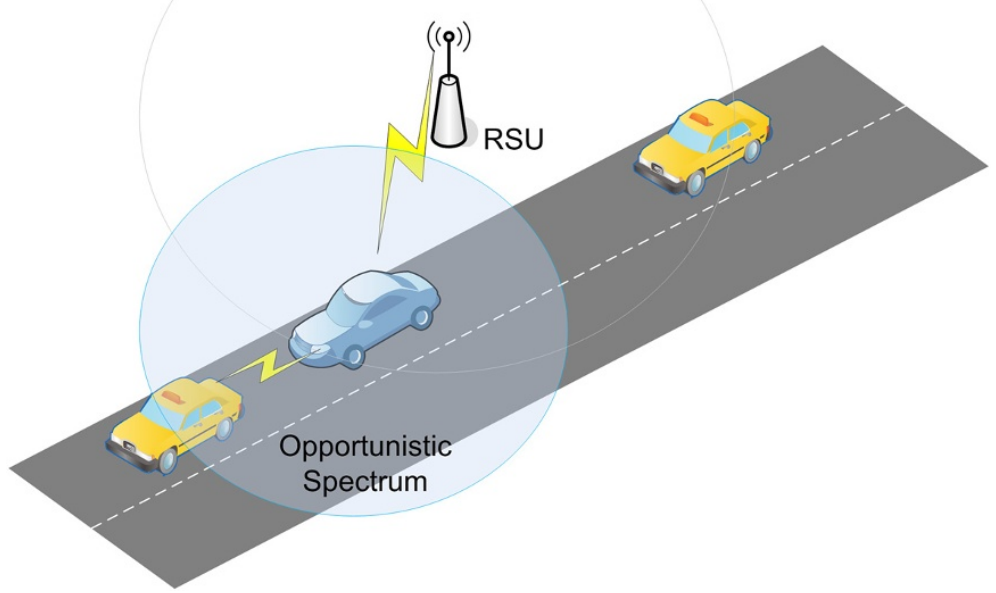

Figure 1 Opportunistic spectrum use for V2V. RSU, roadside unit; DSRC, dedicated short-range communication. 
exchange spectrum information to get information on the spectrum availability. This enables other vehicles to know the spectrum characteristics of the road to be traversed in advance and they can take proactive adaptive measures. In general, CR-VANETs will improve the performance of existing and emerging vehicular applications such as $\mathrm{V} 2 \mathrm{~V}$ communication, entertainment and Information Systems, and public safety communication.

In this paper, we focus on CR-VANETs. Section 2 provides some background and challenges on vehicular networks followed by an overview of cognitive radio technology. Section 3 discusses the motivations of using cognitive radio technology in vehicular networks. A survey of recent advances in CR-VANETs is provided in Section 4, and Section 5 describes some related projects and testbeds. Finally, some open issues which need further attention from the research community are discussed in Section 6 followed by conclusions in Section 7 .

\section{Background}

This section provides some background and challenges on vehicular networks followed by an overview of cognitive radio technology.

\subsection{Vehicular communications}

Modern vehicles are making inroads in the market. These vehicles are not only equipped with global positioning system (GPS) and navigation systems, but also more advanced features such as environmental awareness to prevent vehicle collisions, multimedia systems, and integrated wireless access systems to improve vehicle performance and user experience. In addition, there is much interest in improving the efficiency of vehicular communications. For this purpose, ITS aim at improving safety, reliability, efficiency, and quality of transport infrastructure and vehicles through the use of information and communication technologies (ICT). Additionally, ITS focus on providing sustainable and affordable transportation by designing advanced applications and services to optimize transportation times and energy consumption.

ITS support different communication scenarios including all types of communications in vehicles, between vehicles, as well as between vehicles and roadside infrastructure. Figure 2 shows the taxonomy of vehicular communications. Vehicular communications can be classified into V2V and V2I. V2I further includes vehicle-toroadside (V2R) communication and communication using cellular networks. In $\mathrm{V} 2 \mathrm{~V}$ communications, a vehicular ad hoc network (VANET) is formed among vehicles for exchanging information e.g., safety information. In $\mathrm{V} 2 \mathrm{R}$, information is exchanged between the roadside unit (RSU) and the onboard unit (OBU) of a vehicle. In V2I, information is exchanged between the RSU, or possibly a cellular network, and OBU.

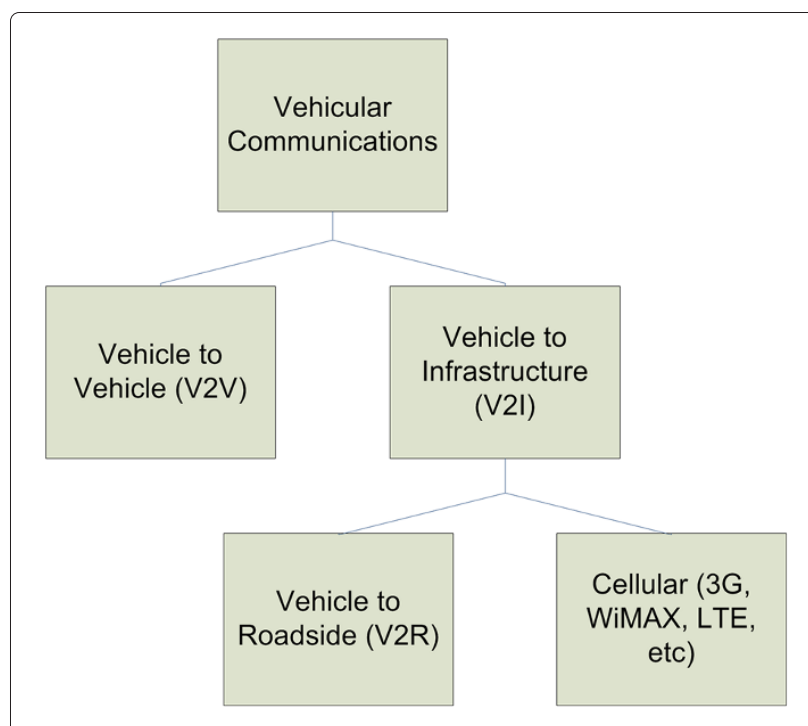

Figure 2 Taxonomy of vehicular communications.

$\mathrm{V} 2 \mathrm{~V}$ communications are relatively more complex and challenging as compared to V2R and V2I communications. The V2I communications are more to exchange information with the centralized servers somewhere in the Internet through various access networks and technologies. Currently, there is continuing effort by cellular network operators to enhance the network capacity that will improve the cellular network support for V2I communications. The V2R communications that are presently deployed are mainly short-distance one-to-one communications, for example, toll collection, whereas the V2V communications are expected to be mostly ad hoc communications with transmission range up to several 100 $\mathrm{m}$. However, currently, the V2V communications are not yet ad hoc-based. There are mainly one-hop communications for unicast and multicast. Standardization bodies and researchers are working on multihop for geo-casting and V2V safety applications, but there are still strong scalability concerns. The transmission range commonly ensured is $300 \mathrm{~m}$ in urban area and about $1 \mathrm{~km}$ in free space.

\subsubsection{Standardization}

The process of standardization for wireless access technology to provide connectivity in VANETs is a work in progress. Older DSRC standards were developed for V2V or V2R for safety and other services, such as fee collection in toll plazas. As mentioned earlier, new DSRC is mostly used as a generic name for short-range, point-to-point communication. It is also used to name the worldwide channels in the $5.9 \mathrm{GHz}$ band, which are reserved for vehicular communications.

Currently, several standards and technologies are available including cellular (2G/3G/4G) technology and IEEE 
802.11p standard that can be used for high-speed vehicular communication. The challenge is to make different technologies and standards interoperable. Cellular $(2 \mathrm{G} / 3 \mathrm{G} / 4 \mathrm{G})$ technology provides good coverage and sufficient security, but it is relatively costly.

The IEEE 802.11p standard will support high-speed vehicular communication at speeds up to $200 \mathrm{~km} / \mathrm{h}$ in $5.9 \mathrm{GHz}$ band, within the USA. It is a link layer technology based on the former IEEE 802.11a amendment. Today, the IEEE 802.11p amendment has been integrated in the new version of IEEE 802.11 standards. It is mainly an adaptation to support efficient infrastructure-less communications (without any prior attachment). In Europe, this technology is promoted by the vehicular industry as a standard for V2V communications and thus, deployment cost is expected to be comparatively less because of largescale production. IEEE 802.11p defines MAC and PHY operations. IEEE also standardized a family of IEEE 1609 standards for WAVE that build on top of IEEE 802.11p. The architecture is shown in Figure 3 and it defines various functionalities related to applications, networking, management, communication, as well as security. A multichannel operation for IEEE 802.11p is also defined which includes a control channel and six service channels. The management plane defines MAC layer management entity (MLME) and physical layer management entity (PLME) for MAC and PHY layers, respectively. For networking, some applications such as infotainment can use TCP/IPv6 or UDP/IPv6, whereas WAVE short message protocol (WSMP) is additionally defined for applications which have strict latency requirements, i.e., safety applications. Nevertheless, IEEE 1609 is not generic, as it is based only on IEEE 802.11p.
The International Standards Organization (ISO) is developing a generic architecture for ITS [14]. Communication access for land mobiles (CALM) refers to a family of standards developed at ISO TC204 Working Group 16 for cooperative ITS. A simplified version of the ITS reference architecture [37] for CALM is shown in Figure 4. Today, different heterogeneous standards exist as a result of silo-application-driven approach. The ITS reference architecture defines a generic protocol stack which aims at enabling convergence. The communication media layer, shown in the figure, considers various media technologies and standards, including cellular (GSM/GPRS, UMTS), older DSRC standards, IEEE 802.11 standards, M5 [38] alias IEEE 802.11p, bluetooth, and infrared. The networking and transport layer considers IPv6, network mobility (NEMO), geo-routing, etc. The facilities layer provides support for application, information, and session. A management plane and a security plane are connected to all the layers for cross-layer functionalities.

Additionally, some harmonization efforts at ISO include the incorporation of IEEE 802.11p and harmonization of CALM-FAST $[39,40]$ (used for short messages having real-time constraints) with IEEE 1609. M5 [38] refers to the adaptation of IEEE 802.11p to the ISO CALM architecture, and G5 [41] is the adaptation of IEEE 802.11p to European constraints by ETSI. Thus, IEEE 802.11p is sometimes referred to as IEEE 802.11p alias M5 alias G5.

\subsubsection{Spectrum policy and regulations}

Several portions of the radio spectrum are regulated by the governments or regulatory bodies for an efficient use of the limited radio spectrum. The increasing use of wireless communications systems dedicated to vehicles will

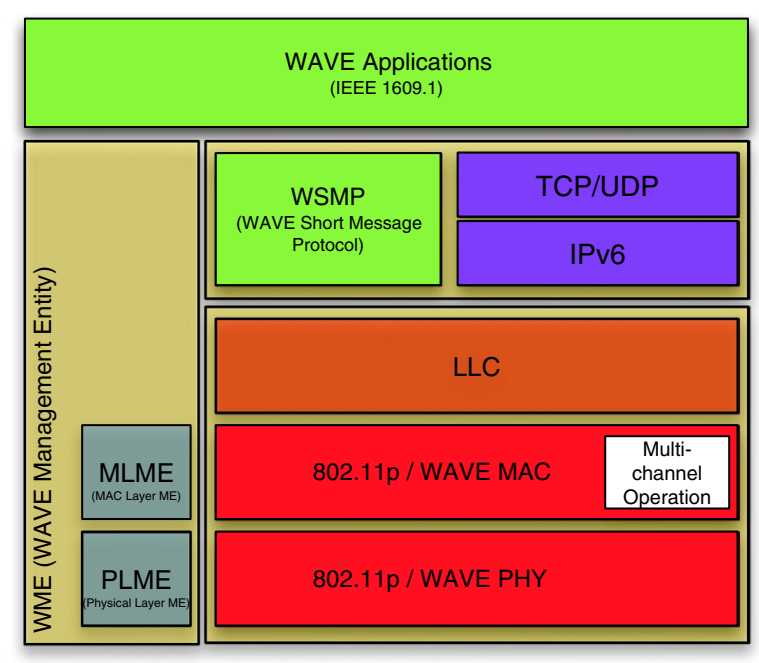

Figure 3 IEEE 1609 WAVE architecture. 


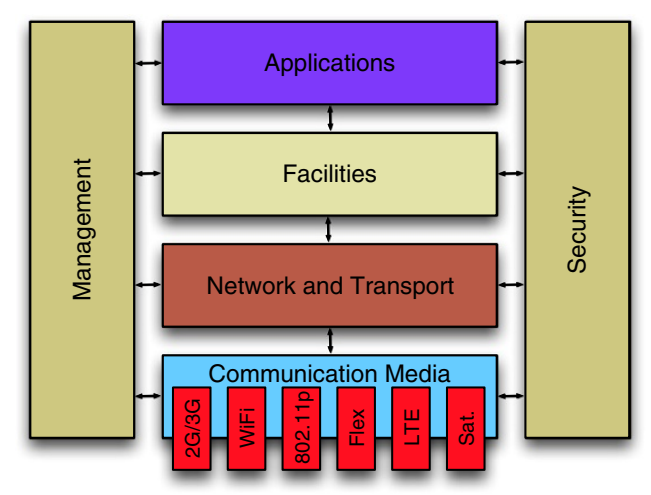

Figure 4 ITS reference architecture.

require spectrum availability for $\mathrm{V} 2 \mathrm{~V}$ communication system. Consequently, FCC has allocated $75 \mathrm{MHz}$ of radio spectrum at $5.9 \mathrm{GHz}$ for V2V and V2I in the USA. However, due to the unavailability of a continuous spectrum of $75 \mathrm{MHz}$ in DSRC band in Europe, Car2Car Communication Consortium (C2C-CC), a nonprofit, industry-driven European organization, has proposed to allocate $2 \times 10$ $\mathrm{MHz}$ for primary use of safety critical applications at 5.9 $\mathrm{GHz}$ range ( 5.875 to $5.925 \mathrm{GHz}$ ). Since this band is used as control channel in the USA, its allocation in Europe will allow for worldwide harmonization.

Recently (since 2012), Japan has allocated $10 \mathrm{MHz}$ in $700 \mathrm{MHz}$ band for ITS because these bands are appropriate for vehicular communications. Radio wave transmission through obstacles is much easier in the $700 \mathrm{MHz}$ bands [42], whereas radio waves in 5.8 to $5.9 \mathrm{GHz}$ are good for short-range communications, e.g., highway toll collection.

Presently, the number of wireless-enabled vehicles is very low and their spectral bandwidth requirements are low as well. However, the increasing number of wirelessenabled vehicles, vehicular communication applications, and high data rate traffic flows will lead to more and more V2V and V2I information exchanges facilitated by wireless communications. ITS will more and more use different wireless access technologies to improve the efficiency and safety of vehicular communication and transportation. In general, different types of ITS depend on radio services for communication and use specially designed technologies. However, if we consider IEEE 802.11p, then it is limited in terms of capacity because it is based on IEEE 802.11a, a quite old version of Wi-Fi (1999).

$\mathrm{V} 2 \mathrm{~V}$ and $\mathrm{V} 2 \mathrm{R}$ are mainly assured by dedicated communication technology in the ITS frequency bands (5.8 to $5.9 \mathrm{GHz}$ ). There is recently a strong pressure on these bands since some companies (Qualcomm, Cisco, etc.) have asked the federal agency to release a part of these bands for more general usages, for example WiFi. Consequently, in the future, these bands could be shared between ITS and other applications, which may lead to crowding of the bands. In addition, the growing demand for vehicular applications (V2V applications) will result in scarcity of spectrum dedicated to vehicle communications. Moreover, vehicle communicationsrelated specific aspects such as high mobility of the vehicles and fast changes in vehicular network topologies add to the challenges in having efficient vehicular communications (Table 1). Therefore, novel techniques and approaches are required to address the problem of spectrum scarcity in VANETs allowing an efficient use of radio spectrum.

With this view, our paper discusses cognitive radio technology for vehicular networks in order to improve vehicular communication efficiency. Cognitive radio techniques can be used in the roadside units (RSUs) as well as the onboard units (OBUs) in a vehicle. $\mathrm{CR}$ will enable them to adapt their transmissions to deal with the fast changes in the radio environment. CR-enabled vehicles will also be able to adaptively select a radio access technology, among many, for communicating with different types of RSUs.

\subsection{VANET challenges}

Here we describe the unique characteristics of VANETs and identify some major issues. Deploying a vehicular networking system requires addressing several challenges posed by the unique characteristics and requirements of vehicular communications [43].

\subsubsection{Mobility and dynamic network topology}

High mobility (for instance, 100 to $200 \mathrm{~km} / \mathrm{h}$ ) of vehicles makes the topology of VANETs very dynamic resulting in very short lived vehicular communication links. Additionally, vehicular density keeps varying from sparse to dense, and high mobility in sparse areas may cause fragmentation problem for VANET, which, in turn, will result in network unreachability for some nodes. Further, high speeds can deteriorate signal due to Doppler and fast fading. These factors can degrade the performance of applications that

\section{Table 1 Challenges and requirements for CR-VANETs}

\begin{tabular}{ll}
\hline Challenges & Required mechanisms \\
\hline Reliable delivery of safety messages & QoS support \\
Highly dynamic topologies & $\begin{array}{l}\text { Algorithms with fast } \\
\text { convergence time }\end{array}$ \\
Scarce spectrum & Efficient use of spectrum \\
Newer and several technologies & Reprogrammability \\
Varied environment and & Machine learning algorithms \\
spectrum availability & \\
\hline
\end{tabular}


have QoS requirements in terms of high reliability, low latency, etc.

\subsubsection{Distributed ad hoc coordination and one-channel vs. multiple-channel paradigm}

In V2I communications, the fixed roadside units can serve as coordinators. However, V2V communications are expected to be self-organizing and to function with or without roadside assisting units. Consequently, authors in [3] argue that one-channel paradigm, with a single shared control channel, is a good solution for V2V communications in the absence of central coordination, considering that various applications will be broadcasting messages to many neighboring vehicles. However, onechannel paradigm comes with the problem of hidden terminal and poses difficult requirements on the design of MAC protocol for $\mathrm{V} 2 \mathrm{~V}$ communications. Though IEEE 802.11 carrier sense multiple access (CSMA)-based $\mathrm{MAC}$ is good for $\mathrm{V} 2 \mathrm{~V}$ communications, its performance degrades in the presence of high number of users.

Moreover, if we reach a larger number of vehicles, the dissemination protocols could lead to a larger overhead. Additionally, a high data traffic density may lead to channel congestion, e.g., in case of an accident and consequent outburst of messages. Multiple-channel paradigm can be a potential solution for such scenarios where instant sharing of message is required between vehicles and thereby reducing congestion on common control channels (CCC). Currently, the approach that is in use is to let all vehicles synchronize to a global time reference and alternate between a common control channel and separate service channels every $100 \mathrm{~ms}$. However, this approach is not efficient [44].

\subsubsection{Routing issues}

Conventional routing protocols are not suitable for VANETs due to their specific network characteristics, e.g., varying network topology and frequent disconnections. Some of the VANETs' routing algorithms can be categorized as opportunistic forwarding [45], trajectory-based forwarding [46], and geographic forwarding [47]. Opportunistic forwarding algorithms (carrying and forwarding a message whenever given the opportunity) are useful in scenarios with frequent disconnections and can be combined with other approaches that use trajectory-based or geographic forwarding. Geographic forwarding algorithms forward packets towards the destination as a function of its geographical location. This routing approach is scalable but not efficient for handling dead ends and voids. Trajectory routing can be the most suitable message forwarding algorithm for VANETs as it considers the road infrastructure as an overlay directed graph, with intersections as graph nodes and roads as graph edges allowing messages to move in predefined trajectories. Moreover, some recent opportunistic approaches for delay-tolerant applications exploit social networking analyses to forward packets. The idea with social-based forwarding is to forward a packet to a node which has a high chance of meeting the destination node in near future.

\subsubsection{Privacy, security, and safety}

Privacy and security issues are highly important in VANETs due to potential threats to traffic flow and human lives by any malicious attempt, for example, fake messages leading to traffic disruption and fatal accidents. Some of the security and privacy issues related to ITS have been discussed in [48-50].

Security protocols for vehicular networks should take into account their specific characteristics such as high mobility and requirements such as trust (vehicles should be able to trust the received messages), resiliency (for interference), and efficiency (e.g., ability to authenticate message in real time). In addition, privacy concerns include preserving anonymity so as to prevent tracking or identification of vehicle for non-trusted parties based on vehicular communication [43]. Nevertheless, such security mechanisms generally come at the cost of degraded communication performance [51].

\subsection{Cognitive radio and software-defined radio}

With the increasing demand for spectral efficiency, cognitive radio has emerged as a very active research area for wireless communications and networks' research community in recent years. CR [26] is an emerging technology that enhances the performance of existing radio by integrating artificial intelligence (AI) with softwaredefined radios (SDRs). Unlike conventional radios in which most of the components are implemented in the hardware, SDRs [24] are radios that use software implementations for some functionalities enabling flexible radio operation. These radios are reconfigurable, and hence the need to modify existing hardware is reduced.

Though several definitions have been provided to describe CR, the commonly used definitions in literature are given by Joseph Mitola and Simon Haykin. Joseph Mitola described CRs [22-24] as intelligent radios that can autonomously make decisions using gathered information about the radio frequency (RF) environment through model-based reasoning and can also learn and plan according to their past experience. Afterwards, Simon Haykin defined CR as an intelligent wireless communication system capable of being aware of its environment, learning, and adaptively changing its operating parameters in real time for providing reliable communication and efficient utilization of the radio spectrum [25]. 
As mentioned previously, a cognitive radio can be built on software-defined radio (SDR) that makes it reconfigurable by converging the two key technologies: softwaredefined radio and artificial intelligence [52-57]. While the field of CR is independent from SDR, CR has its origin in SDR as an enabling technology to support its implementation.

In the context of VANETs, with increasing number of vehicles equipped with wireless communication systems, vehicular communication will require faster connectivity and thus, wireless spectrum will have to be adapted to the new requirements (of bandwidth). Using CR and SDR will prevent the need to implement hardware upgrades with emergence of new protocols in future. SDR enables 'reconfigurable systems' for wireless networks. CR allows SDR to determine which mode of operation and parameters to use. In general, CR and SDR will allow CR-enabled vehicles to search for the best frequency-based predetermined parameters.

\subsubsection{Cognition cycle}

Here we first describe the two main features of CR: cognitive capability and reconfigurability. Then, we briefly discuss the concept of cognition cycle of CR as well as some specifics related to CR-VANETs.

A CR-enabled device adapts its operational parameters as a function of its environment [18]. CR components are mainly radio, sensor, knowledge database, learning engine, optimization tools, and a reasoning engine. CR has cognitive as well as reconfigurability capabilities [25,26,58]. Cognitive capability allows CR to sense and gather information (e.g., different signals and their modulation types, noise, transmission power, etc.) from its environment and, for example, secondary users can identify the best available spectrum. The reconfigurability features of CR allow it to optimally adapt the operational parameters as a function of the sensed information. CR systems involve PU and SU of the spectrum; primary users are license holders, while secondary users seek to opportunistically use the spectrum through CR when the primary users are idle.

The cognition cycle of CR consists of multiple phases: Observe, Analyze, Reason, and Act. The goal is to detect available spectrum, select the best spectrum, select the best operational parameters, coordinate the spectrum access with other users, reconfigure the operational parameters, and vacate the frequency when a primary user appears.

A spectrum hole refers to a portion of spectrum not being used by the primary/licensed user at a particular place and time. It is detected through spectrum sensing and signal detection techniques. The SUs opportunistically access the spectrum if the sensed portion of spectrum is found empty. The secondary user can opportunistically use different spectrum bands by adaptive spectrum switching through spectrum hand-offs. However, the secondary user is responsible to detect arrival of any primary user. If a primary user is detected, then it should vacate the licensed portion of the spectrum in order to prevent interference.

A possible cognition cycle for CR-VANETs in shown in Figure 5 having the four stages: Observe, Analysis, Reasoning, and Act with some customized functionalities for vehicular networks. The Observe stage consists of sensing as well as the consideration of location, policies, and application QoS needs. In the Analysis and Reasoning stages,

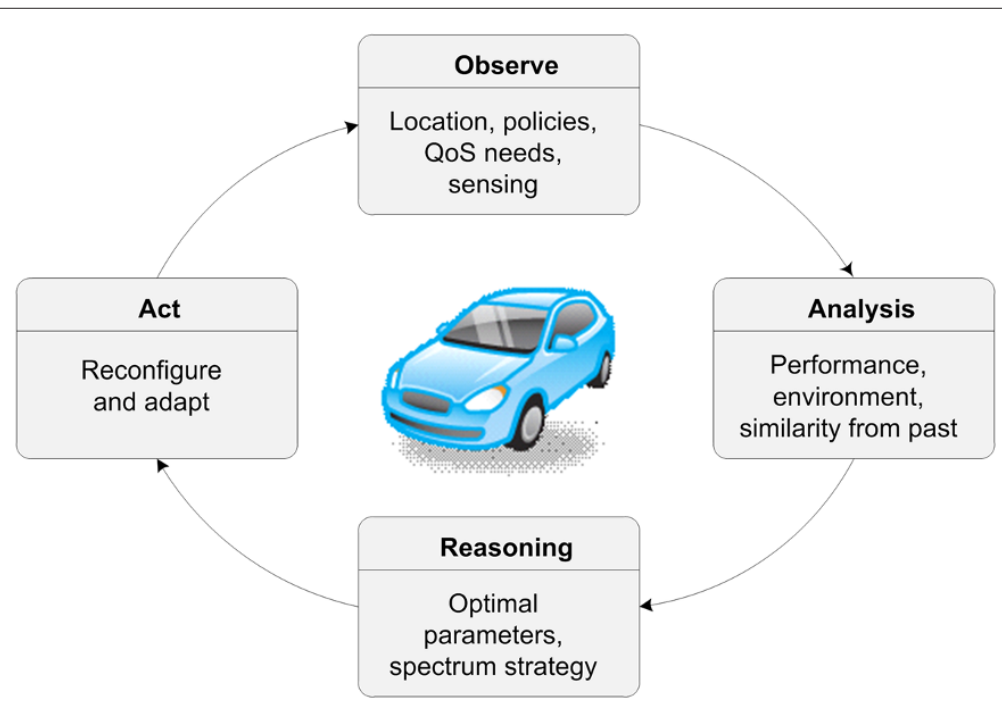

Figure 5 CR-VANETs cognition cycle. 
the system performance and the radio environment are analyzed, similarities from the past are considered, and optimal parameters as well as optimal spectrum strategies are determined. The reconfiguration and optimal adaptation is finally done in the Act stage.

The CR-VANETs cognition cycle has a lot of similarities with that of CR, but some differences are due to the nature of high-mobility vehicular environment. Most of the vehicles will be equipped with navigational systems, and hence location as well as mobility prediction based on the current direction of movement can be used for cognition. The use of knowledge database becomes more relevant as a vehicle may pass through the same location at approximately the same time of the day. Thus, past experiences can be combined with the current location for optimizing the operational parameters. Finally, it should be noted that the requirements for CR-VANETs are more strict in terms of faster adaptability and faster running time of the cognition cycle due to the dynamic nature of the vehicular environment.

\section{Motivations for CR-VANETs}

Currently, high-speed modern vehicles and a large number of cars move on the road every day. ITS [13] aim to develop advanced applications for improving safety and efficiency of road infrastructure and transportation. This will enable users to utilize transport networks in a smarter and safer way.

Presently, the primary objective of vehicle industry is to improve the traveling experience of users by enhancing vehicular communication capabilities with better safety and efficiency, as well as Internet access and infotainment applications.

The number of vehicles on road is always increasing as well as the demand for intervehicle communications. However, ITS and vehicle communications need to overcome several technical challenges. Protocols and applications designed for vehicular communications should consider various important factors such as communication infrastructure, road infrastructure, vehicle density, user demands and types of vehicular networks, as well as available wireless spectrum. Many envisioned applications will need uninterrupted and reliable connectivity and this can be challenging in high-speed vehicular scenarios. New protocols are needed that can deal with rapidly changing environment and are fault tolerant especially for applications related to safety. Overall requirements for such applications are QoS support, adaptability to fast changing environment, robustness, and additional bandwidth to deal with congestion and high bandwidth requirements of some applications such as video streaming. We argue that the cognitive radio technology can help in answering some requirements, and the motivations leading to CR-VANETs are provided in the remaining part of this section.

\subsection{QoS requirements}

QoS support is important for applications such as related to safety. From the point of view of radio technology, satisfying QoS guarantees is easier when there is enough bandwidth, which can be traded with QoS guarantees such as low delay or high reliability. Additionally, there should be mechanisms to protect important flows from lower priority flows. We discuss the issues related to bandwidth and flow priority in the following text.

\subsubsection{Bandwidth scarcity and congestion}

Wireless communication is popular as ever and the demand for more bandwidth and spectrum is ever increasing. In addition, applications like video streaming are becoming popular, which, in turn, consume high bandwidth and can cause congestion. Similar problems of bandwidth scarcity and congestion will impact vehicular communications with growing demands. Recently, many car manufactures have started to provide connectivity features in their cars enabling Internet connectivity through cellular networks (that provide mobile voice and data connections) and other wireless access technologies for accessing various applications. However, the performance of cellular networks suffers in urban areas (large cities) due to congestion of cellular spectrum while in rural areas, cellular coverage is either absent or low. Moreover, as mentioned previously, for dedicated communication, ITS are based on IEEE 802.11p, which is based on an old version of Wi-Fi, namely, IEEE 802.11a, and thus, it has limited capacity. Presently, there is a lack of applications for intervehicle communications, which will change in the future. Car manufacturers look unlikely to buy spectrum. Additionally, note that spectrum is a scarce natural resource and should be used efficiently. Thus, opportunistic spectrum access is an attractive solution.

\subsubsection{Offloading lower priority flows}

Many applications are envisioned for VANETs such as infotainment. Some of these video applications, for example, peer-to-peer (P2P) video sharing and multimedia advertisements can consume a lot of bandwidth and can cause collision with other flows. One idea is to use the main $802.11 \mathrm{p}$ spectrum for important flows and use additional bandwidth available with cognitive radio, opportunistic spectrum (see Figure 1) for lower priority video flows, and P2P traffic.

\subsection{Resiliency}

The flexibility and agility offered by cognitive radio is very useful for resilient communications in vehicular scenarios. 
In case of emergency situations, CR can be reconfigured in real time to operate in emergency mode by focusing on minimizing bit error rate (BER) and avoiding interference [59]. This property is very useful for ITS safety applications, and with CR functionalities, some objectives like bandwidth maximization or power minimization can be flexibly traded for more resiliency.

\subsection{More spectrum holes on highway}

In many cases, highways are open spaces and there is a high chance of finding a spectrum hole that can be accessed opportunistically. This is unlike downtown and urban areas where chances of finding spectrum holes can be low due to high population. In fact, some experiments have been done [29] which show free abundant spectrum available for opportunistic use on highways. Thus, cognitive radio technology is very attractive as it can exploit such spectrum availability using opportunistic spectrum access. This, in turn, can answer some of the bandwidth and congestion problems discussed above.

\subsection{Sufficient space and power supply in vehicles}

Some of the advanced cognitive radio capabilities come at a price in terms of bigger size of onboard units. Some functionalities can also consume energy. However, vehicles have sufficient space and power supply and are not limited by them unlike the case with smart phones and other highly portable devices. Cost can still be a factor, but performance vs. cost trade-off can be exploited. Cost can also be reduced with the help of mass production, by optimally designing an $\mathrm{OBU}$ with cognitive radio capabilities.

\subsection{Reprogrammable vehicular telematics}

Every 2 to 3 years, a new communication standard is being proposed, such as DVB-H, DVB-T2, WiMaX, 802.11p, LTE, and HSDPA. Some of these standards face the risk of becoming a commercial failure such as was the case with DVB-H. Moreover, different countries have different regulations related to spectrum usages, transmit power limits, etc. Thus, global manufacturers of vehicles face a dilemma on which technology to deploy and how to deploy different versions of communication units for different countries. A vehicle has a life cycle of more than 15 years, and onboard communicating devices should not become obsolete during that period. Moreover, the drivers as well as passengers would like to use the newer technologies that come out in the future.

Cognitive radio combined with SDR offers a solution to cope with evolving and numerous technologies. Reconfigurable and reprogrammable capabilities of CR and SDR provide the possibilities to design 'future proof' onboard units that are upgradeable with software updates. This allows flexibility in deploying different versions of units for different countries and allows upgradeability when new technologies come out in future.

An OBU can integrate an SDR transceiver with navigational devices (GPS) and onboard computer to provide capabilities of context awareness and adaptation.

\subsection{Highly mobile environment}

Deploying base stations to provide wireless services (e.g., Internet access) in vehicular networks is challenging due to the highly mobile environment. VANETs are considered as a special case of mobile ad hoc networks (MANETs) due to their specific characteristics such as special mobility pattern, varying vehicle density, and interference with other types of networks.

Several applications of VANETs have been proposed that take into account the above-mentioned constraints of vehicular communication. As described before, different communication technologies have been standardized (WAVE, IEEE 802.11p, etc.) for vehicular communications. However, presently, the deployment and performance evaluation of the proposed standards, especially in a large-scale vehicular environment, are works in progress. Moreover, different standards and protocols proposed for vehicular communication applications should be interoperable. Additionally, short-range communication protocols may not be able to provide good Internet connectivity for high-speed vehicles and that would require developing new efficient protocols suitable for them.

\section{Recent advances in CR-VANETs}

Cognitive radio technology [36] presents a promising solution for addressing the problem of spectrum scarcity. Vehicle communication may benefit from cognitive radio technologies such as dynamic spectrum access, adaptive software-defined radios, and cooperative communications as shown in [60-62]. CR-enabled VANETs can use additional spectrum opportunities in TV bands according to the QoS requests of the applications.

Existing works on CR $[36,58]$ focus on various issues that include investigating techniques for spectrum sensing and spectrum access (dynamic spectrum access), cooperative communications, MAC protocols [63], routing protocols, QoS [64], and software-defined radios. While several studies exist in literature on applying CR to wireless mesh networks, ad hoc networks, and cellular networks, the research on applying CR to VANETs is still in its early stage. The research solutions proposed for general-purpose CR networks cannot be directly applied to CR-VANETs as the unique features of vehicular environment, such as the role of mobility, and the cooperation opportunities need to be taken into account while designing the spectrum management functions for CR-VANETs. Thus, the existing solutions need to be 
customized for CR-VANETs to account for high mobility, dynamic topologies, frequent disconnections, etc. Unlike static CR scenarios, in CR-VANETs, multiple cooperating vehicles (during busy hours) can exchange spectrum information to get information on the spectrum availability. Moreover, this enables adaptive operations and proactive response feasible for the vehicles that follow.

In this section, we present some of the recent works that focus on using cognitive radio technology for vehicular networks. We provide a taxonomy of recent advances in CR-VANETs, in Figure 6. Different approaches are classified into spectrum sensing, spectrum management and QoS, and network. These approaches as well as their sub-categories related to cooperative sensing, sensing in fast varying channels centralized to distributed spectrum management approaches, routing, mobility management, content distribution, etc. are discussed in the following text.

\subsection{Architecture}

The network architecture of CR-VANETs consists of vehicles with onboard units (OBUs) and infrastructure units such as RSUs and base stations of network providers. Note that setting up of roadside units everywhere may be costly; thus, V2V communication, including multihop, should be able to work without any infrastructure support.

Several functionalities are needed for CR-VANETs paradigm, and an initial architecture related to different functionality blocks and protocols is proposed in [65]. The architecture proposed by its authors consists of several blocks related to policies such as those imposed by
FCC and local authorities and onboard sensors, including radio sensors and GPS. It also includes a knowledge database that consists of previously learned cases, rules, spectrum information, and radio maps. Further, it consists of a cognitive engine that is the core of all functionalities. Additionally, there are software-defined radio or multiple radios, performance measurement block for feedback, and finally a user interface to allow users to configure the system offline. Figure 7 illustrates the architecture showing these different functional blocks for CR-VANETs.

As illustrated in the figure, the cognitive engine (CE) uses real-time data from onboard sensors and history and map information from knowledge database to learn from the present as well as the past. It then controls the operation of software-defined radio through optimization of transmission parameters and decision making related to spectrum use and management.

A taxonomy of machine learning approaches for $\mathrm{CE}$ is provided in Figure 8 . The classification is done in terms of supervised learning, decision making and parameter tuning. Supervised learning can be used for prediction or classification, by learning the mapping between the given inputs and outputs. It is called supervised learning because the outputs for the given inputs are known beforehand. For example, in the case of signal identification, the target signal and the input signal features are known during the learning phase. Decision making is related to the problem of making optimal decisions. Some available tools for decision making use reinforcement learning, which can be used in unknown environments, or case-based reasoning based on history, etc. Parameter

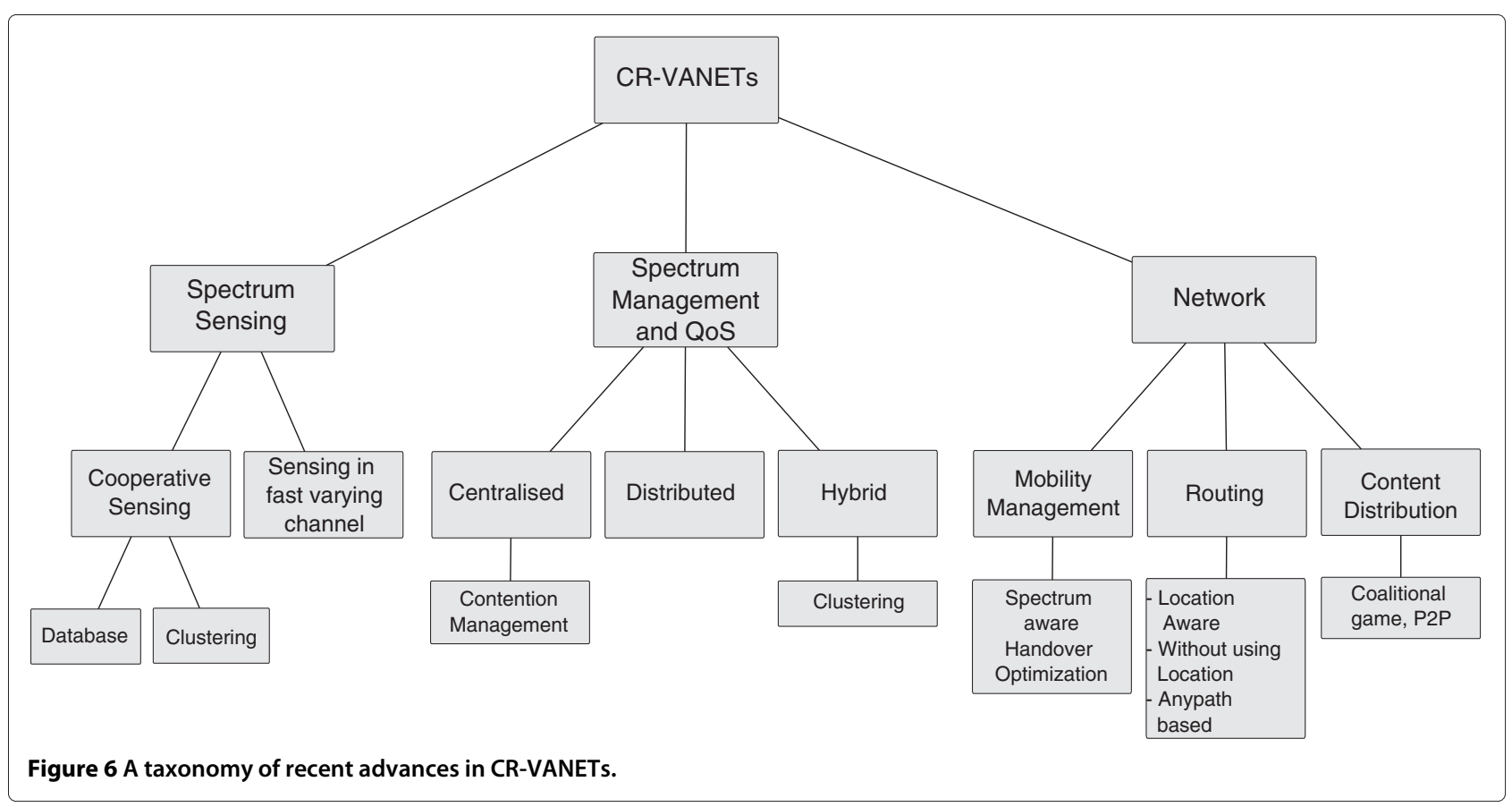




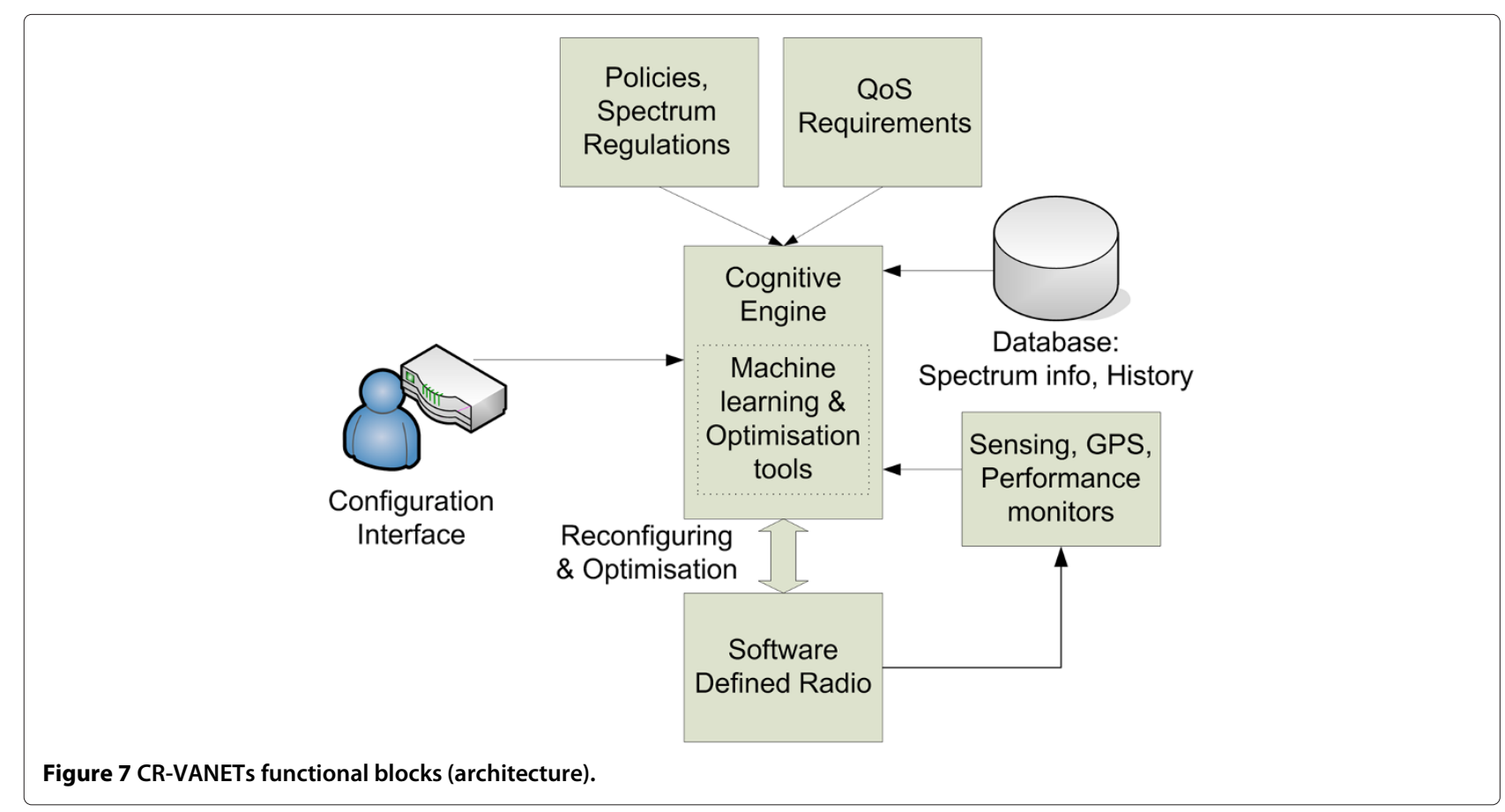

tuning is needed to set the parameters optimally. A system model is used, and some metaheuristics can be used to find the optimal values of the system parameters for a given optimization objective. Multiple conflicting optimization objectives, such as maximization of bandwidth vs. minimization of bit error rate, can be assigned with weights differentially according to the desired mode of communication, ranging from bandwidth maximization mode to emergency mode for high resiliency [59], etc.
Several machine learning techniques have been studied in the literature to realize cognitive functionalities in the general context of cognitive radio. However, they need to be adapted for CR-VANETs scenario. Some interesting ideas for applying machine learning to CR-VANETs are discussed in [61]. We summarize those ideas in Table 2 and discuss them in the following text.

The authors of [61] argue that case-based reasoning is interesting for CR-VANETs scenario because vehicles

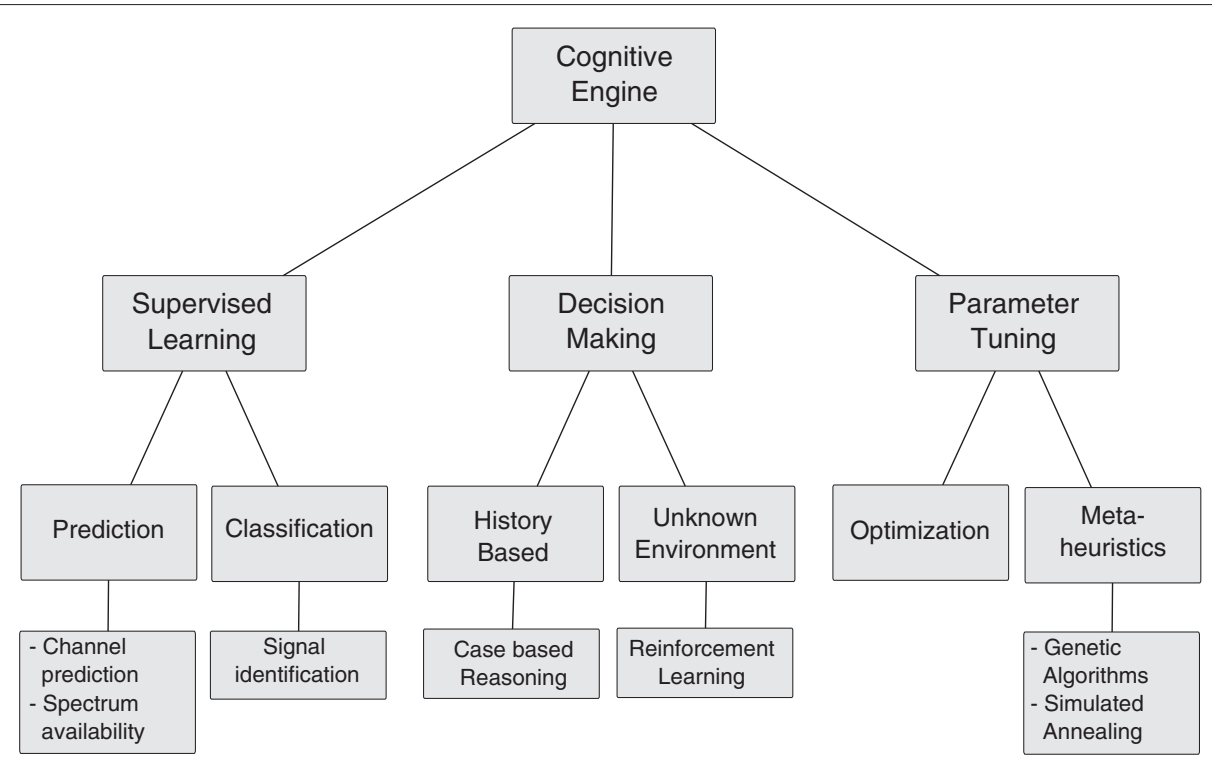

Figure $8 \mathrm{~A}$ taxonomy of machine learning approaches for Cognitive Engine. 
Table 2 Machine learning in CR-VANETs (summarized from [61])

\begin{tabular}{ll}
\hline Machine learning tools & Application \\
\hline Case based reasoning & $\begin{array}{l}\text { Faster convergence from exploiting previous } \\
\text { solutions for example when traveling through } \\
\text { same area on same day of the week }\end{array}$ \\
$\begin{array}{ll}\text { Support vector machine } \\
\text { and neural networks }\end{array}$ & $\begin{array}{l}\text { Signal classification and identifying } \\
\text { spectrum holes, channel prediction } \\
\text { Reinforcement learning }\end{array}$ \\
$\begin{array}{l}\text { Channel selection, real-time learning, can } \\
\text { be used in unknown environments }\end{array}$ \\
$\begin{array}{l}\text { Genetic algorithms and } \\
\text { simulated annealing }\end{array}$ & $\begin{array}{l}\text { Optimization of transceiver } \\
\text { parameters }\end{array}$ \\
\hline
\end{tabular}

have a high chance of passing through the same locations at approximately the same time of the day. This is especially the case for example, for public transports, such as buses and trains but also for cars considering that people commute everyday from their homes to offices, at approximately the same time. With case-based reasoning, the optimization process can be populated from the previously found optimal configurations, and then further search for optimization can start from there. This can highly improve the convergence time of optimization algorithms such as genetic algorithms and simulated annealing, which in turn can be used for optimizing transmission parameters.

Machine learning tools for classification such as support vector machines and neural networks are interesting for signal identification and discovery of spectrum holes. With cognitive radio, it is required that the opportunistic use of spectrum should not collide with primary user traffic. Thus, classification tools can be used to enhance the detection of primary user signals and can provide improved accuracy as compared to just using energy detection. This improved primary user detection accuracy is desired especially in VANETs scenarios, where highmobility conditions leading to signal fading can cause difficulties in spectrum hole detection. Neural networks can also be used as function approximators to predict throughput at a given location on a particular time of the day. Different inputs such as PU and SU traffic statistics can be mapped to predict throughput through trained neural networks [61]. Some online learning tools like reinforcement learning can be used. Such tools are useful in unknown environments and are useful for channel selection and decision making.

\subsection{Spectrum sensing}

Spectrum sensing is required by cognitive radio to detect the presence of spectrum holes and more importantly to detect the presence of PUs. The priority is to avoid interference with PUs and thus, spectrum sensing should be highly reliable.
High mobility in VANETs can deteriorate the performance of spectrum sensing due to signal fading and fast changing location. In the case of fast varying channels over time, some works focus on improving the performance of sensing [66] and signal identification [67-69]. Similarly improved algorithms have been proposed for channel estimation and equalization [70] in high-speed environments.

Additionally, cooperative spectrum sensing [71] can be used to tackle the problems due to high mobility. Several spectrum sensing vehicles combined with a regularly updated central spectrum database can improve the performance of spectrum sensing in VANETs scenarios. The work in [72] investigates the effect of mobility on the performance of spectrum sensing in CR networks. The study confirms that cooperation can improve the spectrum sensing performance as a result of increased spatialtemporal diversity in received primary signal strengths.

This forms the basis of their proposed sensing technique that finds an optimal combination of the number of sensors for cooperation and the number of times to sense the spectrum in order to reduce the sensing overhead. This work does not specifically target CR-VANETs scenario, but it is still interesting for that. Additional works such as $[31,34,73]$ aim to achieve better spectrum sensing precision and efficiency through a coordinated spectrum sensing. The data sensed by the vehicles is sent to RSUs that forward the gathered data further to a central processing unit. The delay and reliability of spectrum sensing in vehicular environments is discussed in [74]. They argue that cooperative spectrum sensing can be used to tackle the sensing limitations of a single vehicle as it exploits spatial diversity. They show that sensing time can be reduced significantly with cooperative sensing as compared to sensing by only a single vehicle.

The authors of [75] point out that cooperative sensing can help a vehicle to detect the spectrum availability in future locations by exploiting sensing information from other cooperating vehicles. An experimental study on sensing performance in VANETs scenario is conducted in [30]. They found that sensing accuracy depends on vehicular speed as well as location. For example, in the areas with open space, the fast fading is not high and hence, sensing performance is good unlike at places such as downtown. They also propose a framework for cooperative spectrum sensing and sharing called Cognitive V2V (Cog-V2V). In Cog-V2V, each vehicle keeps a spectrum availability database that is regularly updated. Note that using such knowledge database, shared by different vehicles or kept distributed among them, is an interesting way of collaborating for spectrum sensing. The data from different cooperating vehicles is merged using weights that depend on distance between the locations at which sensing samples were collected. Intuitively, they 
calculate weights such that weights from nearby vehicles are weighed lower as they are likely to have similar radio conditions due to their similar location. After the spectrum opportunities are detected, Cog-V2V decides the channel to be used between the two vehicles.

Another work [76] uses spectrum database as well as support from infrastructure. They also provide guidelines for the uniform placement of RSUs that will provide information about the availability of TV channels and thereby support the mobile CR-enabled vehicles. Systems using centralized approaches and RSUs can be effective, specifically for safety applications as it improves their reliability by improving data delivery in vehicular networks.

However, the centralized approach is not apt to support multihop communication, especially in the absence of infrastructure support. Nonetheless, in the absence of central infrastructure, clustering techniques can be used to create cluster leaders among several vehicles. Cluster leaders can then assume the role of central entities responsible for coordinating spectrum sensing and data fusion as used in [35].

In [77], a different type of sensing is used that focuses on estimating the amount of congestion in a given channel. The authors address the problem of insufficient spectrum for reliable exchange of safety information over congested urban scenarios through the use of cooperative spectrum sensing provided by vehicles. The spectrum sensing provided by vehicles is used to provide a distributed measure of contention on the roads and locate white channels in the industrial, scientific, and medical (ISM) bands. If contention is detected, free channels from the ISM spectrum are allocated to the vehicles. Note that some of these works are also discussed in the next section in the context of spectrum management.

\subsection{Spectrum management and QoS support}

One of the main focuses of research in CR-VANETs is to design specific spectrum management techniques which take into account spectrum sensing and access as well as mobility for CR-enabled vehicles. Spectrum management should also aim at providing QoS support in CR-VANETs scenario. QoS guarantees are important for messages and signals related to vehicular safety. QoS guarantees can be in terms of maximum permitted delay and reliability in terms of permitted packet loss, etc. For example, in the event of an emergency brake, the vehicles that follow should be notified as quickly as possible in order to leave some time for other drivers to react.

However, providing such QoS guarantees can be difficult in case of congestion or when there are several vehicles. Moreover, it is noteworthy that the feasibility of using TV white space for vehicular communications is restricted due to the scarcity of TV white space in large urban cities, where the average number of vehicles is also higher as compared to the remote areas. Such factors can make it difficult to ensure QoS support for some important applications.

Taking the example of safety messages, authors in [77] argue that the bandwidth of a single DSRC control channel may not be enough for reliable delivery of safety messages during congestion. They propose to use cognitive radio to augment the spectrum of the control channel. They proposed to use TV white space (TVWS) spectrum to enhance the bandwidth of the control channel [15] and in a follow-up work [77], $5.8 \mathrm{GHz}$ ISM band is used instead of TVWS, as $5.8 \mathrm{GHz}$ ISM band is the neighboring band to the DSRC $5.9 \mathrm{GHz}$ band and it can simplify the transceiver design. They argue that Wi-Fi users, who use $5.8 \mathrm{GHz}$ ISM band, are unlikely to be present on highways.

In order to counter congestion problems, authors in [77] first propose a contention metric $C_{r}(t)$ to estimate the amount of congestion in different regions $r$, at time $t$ :

$$
C_{r}(t)=\alpha \frac{D_{r} \times B_{r}}{S_{r}}+\beta U_{r} .
$$

where the delay $D_{r}$ is the delay experienced by the transmitted packet and it increases during congestion. The parameter $B_{r}$ is the channel's bitrate, $S_{r}$ is the average packet size, and $U_{r}$ is the number of untransmitted packets due to congestion. The parameters $\alpha$ and $\beta$ are tuning parameters, which are estimated from curve fitting of simulation data. Different scenarios were simulated to obtain such data by varying the number of vehicles, payload sizes, and bitrates.

Different vehicles perform sensing and report the measurements to their nearest RSU. RSUs communicate with other RSUs and vehicles to form an estimate of congestion in different zones. Based on congestion estimates, a fuzzy-logic-based controller is used to decide the amount of spectrum needed, and RSUs allocate more spectrum to the vehicles traveling in a zone with increased congestion.

The idea is that the spectrum is a scarce resource and additional spectrum is allocated only when needed. Additionally, the proposed contention metric looks interesting and it will be interesting to see how it can be used in other 802.11-like scenarios. Another interesting research direction is to explore improved contention metrics.

Several interesting ideas related to dynamic spectrum access in CR-VANETs are explored in [44]. They call the dynamic spectrum allocation problem for VANETs as vehicular dynamic spectrum access (VDSA). Several VDSA approaches covering spectrum measurements and machine learning are proposed; for example, reinforcement learning is used for intelligent channel selection (also presented in [65]). Moreover, a testbed implementation with some experimental results is also provided. A feasibility analysis is done for VDSA using queuing theory. Different TVWS bands are considered as 
servers which are either occupied by PUs or not. Taking the case of V2I communication, the requests to access those channels from SU vehicles are modeled as clients arriving in a queue and these requests stay in the queue until they are served. Thus, with this formulation, the queuing theory results can be used to analyze VDSA scenario. However, it is pointed out in [78] that such model can be over optimistic because vehicles can move out of RSU range. Thus, vehicular dynamics need to be taken into account and an analysis considering such dynamics is proposed in [78].

Additional works such as in [30,31] propose to first detect the available spectrum resources, then according to the QoS requirements of the vehicular applications, spectrum decision algorithms select the channel to be used at a specific location. Finally, note that even if vehicles have sufficient power supply, the energy consumption is still an important factor for environmental considerations [79]. An energy-efficient technique is proposed in [79] by formulating the problem of deciding whether to use direct transmission or relay-based transmission as an optimization problem, with the objective of minimizing energy subject to QoS constraints in terms of delay.

Most of the above approaches assume a centralized architecture where decisions can be taken in a central manner. However, this may not be always possible in the case of CR-VANETs. Thus, in [35] the authors focus on a clustering strategy as illustrated in Figure 9 where vehicles are grouped into clusters and one of the vehicles act as a cluster leader making central decisions for the cluster. It can be seen as a hybrid of distributed and centralized approaches. The authors study the problem of optimal channel access to provide QoS for data transmission in CR-VANETs. The data from cluster members is first transmitted to the cluster leader and then it is forwarded to its destination.
The problem is to opportunistically use available channels using CR, optimally reserve some bandwidth in DSRC channels, and control the size of the vehicular cluster. The authors use constrained Markov decision process (MDP) for decision making. The vehicles use two radios: one for opportunistic spectrum access and the other for DSRC channel. Vehicles send a request to join a given cluster using DSRC channel. The cluster head takes the decision of whether the new node should be added to the cluster or not. The cluster head also optimally reserves some bandwidth in the DSRC channel. For opportunistic spectrum access, the cluster head collects the sensing results from cluster members and then the scheduling decision is broadcasted to the cluster. The authors point out that there are two different time scales involved in decision making: opportunistic channel access (fast) and cluster size control with DSRC bandwidth reservation (relatively slow). Thus, a hierarchical MDP with two levels with each level corresponding to each problem is used. These two levels interact with each other using the following parameters: QoS performance measures, location, cluster size, and available bandwidth in DSRC channel. The optimization problem is solved using linear programming, and different statistics and variables are assumed to be available in advance. Thus, one interesting extension of this work could be to explore online learning.

Some distributed spectrum management schemes exist for ad hoc CR networks that focus on MAC design [63] as well as QoS [64]. Such proposals may be customized for CR-VANETs. The distributed opportunistic multiChannel MAC (OMC-MAC) proposal in [64] assumes a fixed CCC, transmits beacons at regular intervals for synchronization, and divides the beacon interval into three phases: channel sensing, contention, and data transmission. SUs sense the channel in the first phase and then

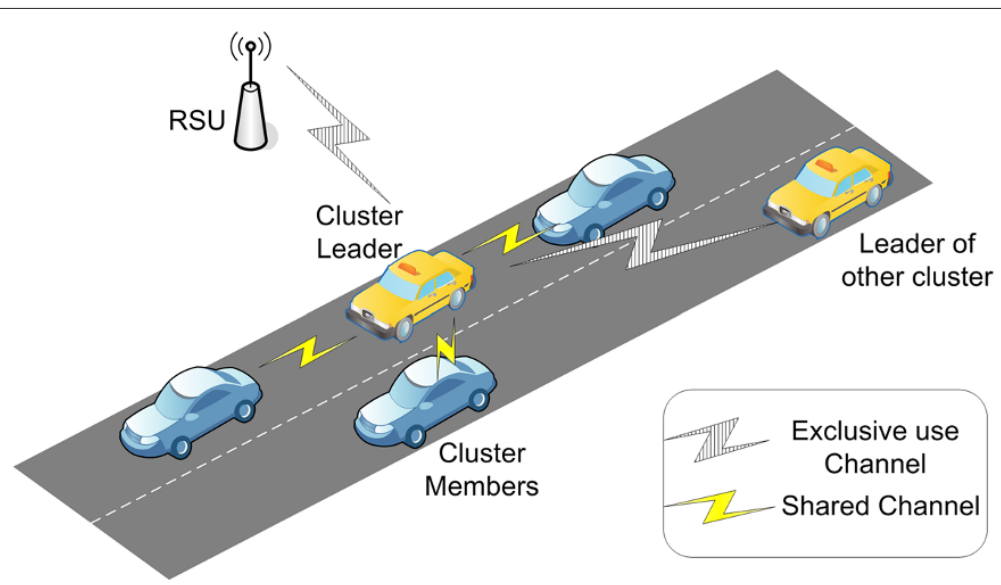

Figure 9 Clustering strategy. Vehicles are grouped into clusters and one of the vehicles act as a cluster leader making central decisions for the cluster. 
using CCC contend for reservations of time slots belonging to data transmission phase. Data transmission phase is then used for contention-free transmission of data based on the reservations on different available channels. QoS provisioning is done by first letting only the prioritized users contend for the reservations and if some time slots are left, then other users can also make some reservations. This can be useful for prioritizing safety messages in CR-VANETs. However, the impact of vehicular mobility and short-lived links needs to be studied for such schemes. The common control channel may also get congested when there are many vehicles. The discovery process of available channels, during the sensing phase, can be improved by using a common spectrum database, as explained in Section 4.2. Adaptive bitrate as well as beacon interval can be explored to further improve the performance.

\subsection{Network layer: routing, mobility management, and content distribution}

In this section, we discuss the works on CR-VANETs that are related to upper layers. We mainly focus on network layer but also talk about P2P approaches that work on top of an overlay network.

\subsubsection{Routing}

Several cognitive routing schemes have been proposed for CR-VANETs, accounting for spectrum scarcity, interference, and high mobility. Some important metrics for routing in CR-VANETs are PU activities, availability of spectrum, geographic location, channel switching delays, hop count, etc.

One initial protocol called Search [80] is proposed for cognitive radio ad hoc networks that is interesting for CR-VANETs scenario also. It selects a route and the channel that avoid the regions of PU activities. It sends route request packets (RREQs) and they reach the destination via different paths. The destination selects the optimal paths based on geographic forwarding. The cost of switching a channel is also taken into account because with cognitive radio the intermediate nodes might have to switch a channel to forward a packet on a given path. Then the paths are combined to minimize the number of hops. Another recent approach based on RREQ packets is proposed in [81] but without using the location information as all vehicles may not have GPS information. Two routing schemes are proposed: (1) First scheme searches through several routes with each route evolving on a single channel; (2) Second scheme discovers only one route, but it exploits several channels.

A recent protocol CoRoute [33] is an anypath vehicular routing protocol for CR-VANETs that exploits geo-location and sensed channel information. CoRoute extends the anypath multirate scheme proposed in [82] to include cognitive radio and geo-location. With anypath multirate routing, a node broadcasts to a set of nodes using a selected rate. Then, any one of them forwards the packet towards the destination in the same way while the other nodes in the set avoid broadcasting duplicate copies if they hear the first broadcast. This routing has an anypath property that any node that received the packet can forward it and hence, the path can change depending on which node received the packet. If multiple nodes receive broadcast then, the one with highest priority forwards the packet. This priority is chosen based on the expected anypath transmission time (EATT), which is the expected time to transmit a packet to the forwarding set depending on packet size, bitrate, and the delivery probability that the packet is received at least by a single node in the forwarding set.

CoRoute extends the anypath multirate routing protocol such that the broadcasts are done on different channels depending on the sensed channel information and geolocation. The set of forwarders is chosen such that the nodes in the set are geographically closer to the destination. The channel with the least amount of congestion is chosen. The priority of the nodes in the set of forwarders is set based on EATT as described before.

\subsubsection{Mobility management}

Mobility management is important for ensuring connectivity even when the vehicles move. The Internet Engineering Task Force (IETF) has defined some IP mobility support protocols that will be used for vehicular networks. For instance, NEMO [83] protocol based on Mobile IPv6 (MIPv6) [84] is being considered for vehicular scenarios. In addition, Proxy Mobile IPv6 (PMIPv6)based NEMO protocol is also a possible candidate [85-87]. Currently, Distributed Mobility Management (DMM) and data offloading techniques are being considered [88]. DMM can reduce the overall path latency and can eliminate the single bottleneck problem of the centralized architectures. Moreover, data offloading is useful for adaptively offloading some flows to other available networks and benefiting from additional available bandwidth.

These mobility management approaches can benefit from the information available from cognitive radio modules. Network selection and handovers can be optimized, using cross-layer optimization, for example, by considering geo-location and spectrum availability to store different signal thresholds used for handover. The spectrum can be allocated among RSUs so as to maximize the coverage and minimize the number of handovers, etc. Additionally, some flows can be adaptively offloaded to the cognitive radio network using data offloading techniques.

Very few works [89] discuss mobility management in the context of cognitive radios. In [90], a joint mobility 
and spectrum management scheme is studied focusing on cellular networks, but the ideas are also interesting for CR-VANETs.

\subsubsection{Content distribution using peer-to-peer techniques} For content distribution in CR-VANETs, one proposal [91] uses V2I communication for content distribution and uses $\mathrm{V} 2 \mathrm{~V}$ communication with cognitive radio to exchange the missing packets. They use a P2P-like content sharing approach using cognitive radio. For P2P communication in the context of CR-VANETs, the problem is to determine that the onboard unit should download which chunk, from which peer, depending on the link and spectrum characteristics. The authors propose an approach based on coalitional graph game. When some vehicles pass a RSU, then they can download some data chunks from RSU and other chunks from peer vehicles. For opportunistic spectrum use, each vehicle senses a set of spectrum channels for detecting the presence of primary users. A channel is chosen which has maximum transmission capacity and is not occupied by primary users or neighboring vehicles.

A utility function is used such that payoffs are a function of the number of successful packets transmitted as well as received multiplied by the probability of successful transmission. The probability of successful transmission is computed by the probability that no neighbor of a given vehicle is transmitting on a particular channel multiplied by the probability that the given transmission does not collide with primary user. The latter depends on the probability of missing the primary traffic and probability of false alarm. The cost of using a link between two vehicles is defined as a weighted function of the total number of neighbors of receiving and transmitting vehicles. This cost function is used to represent the probability of data collision because any transmission on this link has a chance of interfering with those neighbors.

Finally, a myopic distributed algorithm is proposed whose objective is to converge towards a graph describing which vehicle connects with which other vehicle or RSU. The proposed algorithm is myopic as it uses only local information, as compared to centralized algorithms which need global information. Initial approach is that each vehicle chooses a graph that just optimizes its own utility based on current neighbors and channel characteristics.

Usually, it is desired for any distributed algorithm to converge. However, authors state that it is difficult to prove the convergence of such algorithms due to dynamically evolving topologies in VANETs scenarios. Moreover, the proposed algorithm can have a ping pong effect that is to oscillate from one graph formation strategy to another. Thus, they introduce a notion of history function which keeps a count of how many times a given strategy was used. If a strategy was already used up to a certain threshold, then it is not used again. With this history function, the authors show that their algorithm converges within a finite number of iterations. The simulation results show that their algorithm can improve the throughput by $250 \%$ as compared to just V2I broadcast.

It would be interesting to see how this work can be extended for the case of multihop communication (only line of site neighbors are used) and selfish users. Additional ways of ensuring convergence could be explored with newer algorithms. Moreover, it would be interesting to consider more detailed link models as presently only a simple model for link layer collisions and contention is used.

\section{Projects and testbeds}

The research on cognitive-radio-enabled vehicular networks is still in its preliminary stage, and there are not many related experimental platforms due to their complex setup and requirements. In this section, we describe some existing projects and testbeds related to cognitive-radioenabled vehicular networks.

\subsection{CORRIDOR}

Cognitive Radio for Railway Through Dynamic and Opportunistic Spectrum Reuse (CORRIDOR) [92] is a French research project that targets opportunistic spectrum access for railways. Communication demands are increasing for modern railways from the point of view of railway operations as well as for providing Internet connectivity to the passengers. However, there is no single universal wireless technology that can answer the needs of heterogeneous services and railway applications. Modern railway applications and passenger's Internet connectivity demands call for more bandwidth and spectrum.

One answer to such needs is cognitive radio. Thus, CORRIDOR plans to use CR technology to support multiple railway applications such as command and control, CCTV, maintenance, and Internet connectivity for passengers. The project aims to develop algorithms and technology for very high speed environments that are typical of railways. The focus will be on spectrum access and management, exploiting TVWS, cognitive engine, crosslayer optimization, mobility management, and handover optimization. Different techniques will be validated with some real on-site trials.

\subsection{PROTON-PLATA project}

The PROTON-PLATA project [93] is a European project that aims at developing a reconfigurable prototype based on emerging software-defined technologies for telematics applications for cars-to-roadside and car-to-car communications. The project proposes a multitechnology cooperative Advanced Driver Assistance System (ADAS) 
that is based on the integration of SDR devices in vehicles.

The first part of the project deals with developing a prototype including SDR device and driver assistance applications to demonstrate the utility of a cooperative ADAS for improving communication performance. This involves designing a multitechnology communication infrastructure and equipping onboard units with SDR devices that support different communication scenarios i.e., V2V communications, V2I communications, and broadcasting (infrastructure-to-vehicle) at the same time. The performance evaluation using network simulation tools forms the second part of the project.

\subsection{Rail-CR project}

The Rail-CR project [94] is a US project that is a part of the efforts toward implementing the positive train control (PTC) technology to equip trains with wireless communication capabilities. This will allow trains to communicate with wayside wireless stations, while moving, and provide useful information related to their location, speed, direction, etc. The project adapts Virginia Tech cognitive radio technology [95] to improve safety and operations of the railways.

The complex radio characteristics faced during train transportation such as continuously changing situation of the train as well as continuously changing communications environment due to varying noise, multiple sources of interference, multiple users competing for scarce spectrum, etc. make wireless communication with trains very demanding. This has motivated the railway industry to use SDRs equipped with a reconfigurable platform for packet-data transmission. Though SDR provides interoperability and enables multiple configurations, it lacks the capability of integrating additional adaptation to alleviate crowded spectrum, intentional jamming, or learning from past experiences.

In this context, the project developed a railroad-specific cognitive radio namely, Rail-CR, capable of fulfilling the requirements of future wireless communication systems for trains. The Rail-CR combines AI-based decisionmaking and learning algorithms with SDR to address the railway transportations' specific need for adaptable communications. The Rail-CR cognitive radio will enhance the performance of railway communications in terms of interoperability, robustness, reliability, and spectral efficiency, and will make it cost-effective to deploy and maintain.

The Rail-CR system includes a CE that relies on the tunable radio parameters (knobs) and observable performance indicators (meters) of the SDR platform. Based on situational awareness from the radio in the form of observable parameters (meters), a cognitive engine uses software-based decision-making and learning algorithms to decide if the radio parameters (knobs) should be adapted depending on sets of predefined objectives. The Rail-CR was evaluated under different interference conditions, and results show that the cognitive engine can manage to overcome the interference by adapting configurable parameters whereas a radio minus cognition capability was not able to overcome interference resulting in high errors or a loss of connectivity.

Further, a case study showing the use of metacognitive radio to improve wireless communication systems used for signaling and train control in railroads is presented in [96]. In a metacognitive engine [96], a master process monitors and adapts the cognition process (i.e., cognitive engine). Metacognition enhances the performance of a cognitive radio with better learning and decision-making capabilities.

\subsection{The cognitive cars testbed}

Research in VANETs has focused on simulation studies to evaluate the performance of communication protocols for VANETs. Testing and evaluation of VANETs communication protocols (e.g., VANETs-based multihop communication system) in real testbed scenarios for example, on the road, has been considered a difficult task as there are virtually no highly dense vehicular testbed that exists. Recently, a new cognitive approach to VANETs research was introduced in [97] that enables performing real vehicular experiments, which usually require several vehicles, using only a few vehicles equipped with wireless communication interfaces. This is done by setting up a virtual overlay network consisting of relaying and interfering vehicles on top of a group of only a few vehicles to conduct experiments. The communication packets travel over a random number of hops and experience interference from random number of vehicle transmitters with varying transmission characteristics.

The cognitive car testbed [98] is based on guidelines presented in [97] to conduct real experiments with VANET applications and communication protocols under constraints of vehicular environment and computing resources. The objective of the cognitive car testbed is to explore the possibility of using cognitive network technologies in advanced vehicular testbeds. It focuses on using cognitive radios to study the impact of different frequency bands and different frequency switching delays on the performance of VANET communication protocols. More specifically, cognitive car testbed enables evaluating a communication protocol vs. different variables including number of hops, density of interfering neighbors, wireless channel conditions, transmission frequency, and switching delay at the node.

In this context, it introduces the architecture guidelines for implementing a VANET testbed and, in addition, provides preliminary results for the experiments conducted 
with an accident warning system developed for highways and a cognitive network based on the software radio (SORA) technology provided by Microsoft. Preliminary results show that cognitive interfaces can be used as an additional tunable dimension in an experiment platform where highly dense vehicular testbeds can be set up with just few real vehicular resources (e.g., few cars and drivers). The cognitive interfaces enable testing novel techniques designed for addressing radio spectrum scarcity in a vehicular environment as well as performance of VANET communication protocols. The performance of VANET communication protocol can be evaluated by tuning two additional variables: frequency band and switching time. The first variable is the frequency band used for transmitting and receiving communication packets and the second variable is the switching time needed to change from one frequency to another.

\subsection{Virginia Tech CR network testbed (VT-CORNET)}

VT-CORNET [95] is an open-source cognitive radio network testbed, developed by Virginia Tech (Blacksburg, VA, USA) for the evaluation of CR wireless communication protocols and applications. The testbed consists of 48 software-defined radio nodes (USRP2-based nodes) deployed over the four floors of a building and equipped with a custom-made daughterboard covering the frequency range of $100 \mathrm{MHz}$ to $4 \mathrm{GHz}$. The CORNET nodes are remotely accessible to the registered users. A unique feature of the testbed is that it uses Software Communications Architecture (SCA) framework that assumes USRP nodes at the physical layer and provides support for generating and visualizing a range of radio configurations.

The testbed focuses on CE design, self-organizing networks, and topology research. VT-CORNET is a highly reconfigurable testbed that enables testing of independently developed CR engines, sensing techniques, applications, protocols, performance metrics, and algorithms. In addition to the static 48 nodes deployed in the ceiling, low-power mobile nodes will also be available to support vehicular environment. The rail-CR project has adapted the VR-CORNET cognitive radio technology for railways as described in the first part of this section.

The CORNET testbed is openly available for conducting research on advanced $\mathrm{CR}$ networks. It provides a collection of resources to researchers lacking the ability to perform advanced experiments because of limited exposure to software-defined radio and CR platforms. The resources are made available to the researchers through flexible RF front ends, open-source software, and FCC experimental licenses.

\subsection{ORBIT testbed}

The ORBIT testbed [99] is an open-access research testbed for next generation wireless networking that allows large-scale reproducible experimentation on next generation protocols and applications. It consists of an indoor radio grid emulator for large-scale reproducible experiments and an outdoor field trial system for supporting real-world experimentation and evaluations.

A 400-node radio grid emulator has been deployed at the WINLAB Tech Center building and is open for general use by the research community to conduct experiments. The ORBIT testbed provides support for conducting a wide range of experiments including mobile ad hoc networks, dynamic spectrum coordination, network virtualization, wireless security, and vehicular networking. The radio grid testbed enables emulation of real-world network topologies via noise injection and packet filtering. The testbed has been recently upgraded to include programmable software-defined radios (GNU USRP/USRP2) for flexible MAC/PHY experiments.

The testbed users can access the radio grid through a web portal to specify their experiments and measurements. As mentioned previously, the testbed can be accessed worldwide for conducting experiments and evaluate protocols and applications related to ad hoc routing, P2P, spectrum sensing, and dynamic spectrum access. Recently, the testbed has started providing support for vehicular mobility as well. The ORBIT management software has been extended to enable mobile vehicular (vehicle-to-vehicle networks) experiments, and the radio grid is now supplemented by a number of outdoor and vehicular nodes.

\section{Open research issues}

CR-VANETs is a relatively new research area and hence, there are many issues that are open to further research. In general, customizing existing CR approaches to CRVANETs is an interesting research direction. Additionally, there are some specific issues and we discuss some of them in this section.

\subsection{Vehicular mobility}

Initial studies $[30,72]$ on the effect of vehicular mobility on spectrum management show that the primary users may have to face adverse interference due to the incorrect detection of occupied frequencies as a result of the Doppler spread generated by vehicular mobility. At the same time, since a CR-enabled vehicle can gather samples of signal at different locations along its path while moving, this increases the spatio-temporal diversity of the samples and, in turn, decreases the chances of inaccurate decisions due to shadowing effects.

Open issues on how the performance of spectrum sensing is affected by vehicular mobility that need investigation include the following: techniques to achieve a balance between cooperation and spectrum scheduling for a CRenabled vehicle on the move, how to use predictable 
mobility of vehicles to improve spectrum awareness, and how the mobility-related parameters such as speed and direction affect the performance of spectrum sensing [28].

\subsection{Vehicle-to-vehicle communications}

Mobile ad hoc networking is difficult in general. In case of CR-VANETs, V2V communication is more complex and difficult because of dynamically evolving topologies and frequent disconnections that are due to vehicles going out of range. This happens more often when the vehicular density is low. Such disconnections can create instability and performance issues for upper layer protocols such as routing and transport (TCP). Some cross-layer strategies to optimize transport protocols, for example TCP, can also be explored such as in [100,101]. For routing in vehicular networks, some approaches based on delaytolerant networking (DTN) are becoming popular. DTN approaches can help in improving the stability and performance of routing protocols by providing some tolerance to disruptions. Thus, CR-VANETs routing protocols also need to integrate some DTN elements to counter frequent disconnections, apart from being spectrum aware.

\subsection{Machine learning and environment characterization in CR-VANETs}

Several machine learning approaches have been applied for cognitive radio. However, as discussed in this paper, CR-VANETs can have more stringent requirements in terms of requiring fast algorithms due to fast changing environment. Moreover, there can be different types of redundancies that can be exploited such as redundancy in network statistics at a given location at the same time of a day. This is especially pertinent in the case of public transport such as buses and trains. Thus, machine learning algorithms that are more specific to CR-VANETs should be explored. Moreover, it is also important to design the knowledge database, which is consultable by machine learning algorithms for faster convergence. Some design issues are the following: which statistics to measure and store, how to divide the region into different zones, how to characterize different environments from the point of view of radio propagation (open space vs. city landscape), how to fuse different measurements, etc.

\subsection{Distributed algorithms}

Many of the works assume centralized decision making for spectrum access and sharing. This may be valid in cases with infrastructure support for example, when a RSU can take central decisions. However, setting up of infrastructure is difficult and using the services of cellular networks has some costs as well as bandwidth limitations. Thus, infrastructure support may not be available all the time and consequently, distributed algorithms are highly desired for CR-VANETs. Spectrum-aware distributed algorithms for CR-VANETs should be explored that only use local information, such as that available from neighboring vehicles. These algorithms should have low convergence time, incur low signaling overhead, and should be fault tolerant.

\subsection{Adaptive beamforming}

Directional antennas are an interesting way to reuse space through beamforming, by focusing the antenna pattern on a specific region. A recent work on analyzing multicast capacity with beamforming is [102]. Beamforming also improves throughput as signal quality is improved in the concentrated beam. Moreover, beamforming is more relevant in vehicles than as compared to, for example, smart phones. This is because smart phones rapidly change their orientation in our hands, which makes it difficult to exploit beamforming with smart phones. On the other hand, vehicles change their orientation only while changing the route and it can be used to enhance the communication. With adaptive beamforming, a vehicle can focus the antenna beam only on a particular vehicle and can thus avoid interference in another zones containing PUs or other SUs. Thus, adaptive beamforming combined with opportunistic spectrum access is an interesting direction to explore.

\subsection{QoE aware optimization}

Most of the works focus on optimizing QoS metrics, such as throughput and delay. Some works optimize user utilities based on generic utility functions. However, it is ultimately the quality of experience (QoE) which matters to the users. For example, perceptual quality, an important QoE component for videos, is important for passengers streaming a video. For video streaming applications, it is now easier to adapt to changing network characteristics with some new standards such as scalable video coding (SVC) and dynamic HTTP streaming (DASH). One of the optimization approaches, in wireless networks, can be to use subjective testing to design perceptual video quality models for SVC and DASH $[103,104]$ and apply such models for optimizing QoE, as done for wireless multicast in [105]. Thus, similar QoE aware approaches can be explored in the context of CR-VANETs by considering additional parameters related to spectrum characteristics and availabilities.

\subsection{CR-VANETs experiment and simulation platforms}

Typically, the hardware experiment platforms designed for VANETs and CR-VANETs consist of regular vehicles and software-defined radios [106,107] and it is complex to set up such platforms involving vehicle mechanics integrated with radios. Thus, currently, simulators are used. Using traffic simulators such as SUMO [108] and network simulators (NS-2/3 and OMNeT ++ ) together enable 
designing simulators that combine vehicle mobility and communication networks. However, there is a lack of suitable simulators with features such as spectrum database, spectrum sensing and management, power spectral density, wireless interference level, and Doppler effects in order to be able to model and simulate varying topology of CR-VANETs and local wireless networks.

\subsection{Security and privacy}

With the use of cognitive radio for vehicular networks, there are additional security-related issues which need to be addressed, such as protecting the privacy of cooperating vehicles and identifying malicious CRenabled vehicles that broadcast false spectrum sensing reports while on the move. Addressing security issues in CR-VANETs is challenging as neighboring vehicles can keep changing over time while moving. While moving on the road, it is difficult to detect a malicious car that may be transmitting fake spectrum sensing reports as this requires rapid detection and correction.

oreover, it is important to consider the privacy concerns of end users while using collaborative spectrum sensing in CR-enabled vehicles. Security issues should focus on how to prevent a potential attacker from tracking a driver's identity and movements by eavesdropping the periodic spectrum information broadcast by the vehicle [28].

\section{Conclusions}

This paper has surveyed novel approaches and discussed research challenges related to the use of cognitive radio technology for VANETs. Though CR deployment in vehicular networks is still in the preliminary stage, CR-VANETs has the potential of becoming a killer CR application in the future due to a huge consumer market for vehicular communications. However, the research solutions proposed for general-purpose $\mathrm{CR}$ networks cannot be directly applied to CR-VANETs due to their unique features that need to be considered while designing the spectrum management functions for CR-VANETs. In this context, several challenges and requirements for CR-VANETs have been identified. We have provided recent advances and open research directions on applying cognitive radio for vehicular networks focusing on architecture, machine learning, cooperation, reprogrammability, and spectrum management as well as QoE optimization for infotainment applications. A taxonomy of recent advances in CR-VANETs is also provided. In addition, some existing testbeds and research projects related to CR-VANETs have been described.

\section{Competing interests}

The authors declare that they have no competing interests.

\section{Acknowledgements}

This work was supported by the CORRIDOR project (ANR-11-VPTT-004) of the French national research organization (ANR). We also thank the anonymous reviewers and Remi Painchault, from Thales, for their valuable comments which helped in improving this article.

\section{Author details}

${ }^{1}$ Department RSM, Telecom Bretagne, 35510 Cesson Sevigne, France. ${ }^{2}$ INRIA Lille - Nord Europe, Univ. Lille 1, CNRS UMR 8022, 59650 Lille, France.

Received: 17 December 2013 Accepted: 10 March 2014

Published: 28 March 2014

\section{References}

1. P Rawat, K Deep Singh, H Chaouchi, J-M Bonnin, Wireless sensor networks: a survey on recent developments and potential synergies. J. Supercomput, 1-48 (2013). doi:10.1007/s11227-013-1021-9

2. Y Toor, P Muhlethaler, A Laouiti, Vehicle ad hoc networks: applications and related technical issues. IEEE Commun. Surv. Tutor. 10(3), 74-88 (2008)

3. H Hartenstein, KP Laberteaux, A tutorial survey on vehicular ad hoc networks. IEEE Commun. Mag. 46(6), 164-171 (2008)

4. A Boukerche, HA Oliveira, EF Nakamura, AA Loureiro, Vehicular ad hoc networks: a new challenge for localization-based systems. Comput. Commun. 31(12), 2838-2849 (2008)

5. F Li, Y Wang, Routing in vehicular ad hoc networks: a survey. IEEE Veh. Tech. Mag. 2(2), 12-22 (2007)

6. J Luo, J-P Hubaux, A survey of research in inter-vehicle communications, in Embedded Security in Cars (Springer, Berlin Heidelberg, 2006), pp. 111-122

7. C Cseh, Architecture of the dedicated short-range communications (DSRC) protocol, in Vehicular Technology Conference, 1998. VTC 98. 48th IEEE, vol. 3 (IEEE, 1998, Ottawa, 18-21 May 1998), pp. 2095-2099

8. J Zhu, S Roy, MAC for dedicated short range communications in intelligent transport system. IEEE Commun. Mag. 41(12), 60-67 (2003)

9. L Cheng, BE Henty, DD Stancil, F Bai, P Mudalige, Mobile vehicle-tovehicle narrow-band channel measurement and characterization of the $5.9 \mathrm{GHz}$ dedicated short range communication (DSRC) frequency band. IEEE J. Select. Areas Commun. 25(8), 1501-1516 (2007)

10. JB Kenney, Dedicated short-range communications (DSRC) standards in the United States. Proc. IEEE. 99(7), 1162-1182 (2011)

11. Amendment 6: Wireless Access in Vehicular Environments (WAVE), Part 11, IEEE Standard 802.11p, IEEE Standard 802.11p. http://standards.ieee. org/findstds/standard/802.11p-2010.html

12. D Jiang, L Delgrossi, IEEE 802.11 p: Towards an international standard for wireless access in vehicular environments, in IEEE Vehicular Technology Conference 2008. VTC Spring 2008 (Singapore, 11-14 May 2008), pp. 2036-2040

13. MA Regan, J Oxley, S Godley, C Tingvall, Intelligent transport systems: safety and human factors issues, no. 01/01 (Royal Automobile Club of Victoria (RACV) Ltd., 2001), Monash University, Australia, 2001

14. ETSI-Intelligent Transport. Intelligent Transport Systems. http://www. etsi.org/technologies-clusters/technologies/intelligent-transport. Accessed on 11 March 2014

15. AJ Ghandour, K Fawaz, H Artail, Data delivery guarantees in congested vehicular ad hoc networks using cognitive networks, in 2011 7th International IEEE Wireless Communications and Mobile Computing Conference (IWCMC) (Istanbul, 4-8 July 2011), pp. 871-876

16. Federal Communications Commission, Spectrum Policy Task Report of the Spectrum Efficiency Working Group (2002)

17. MA McHenry, NSF spectrum occupancy measurements project summary. Shared spectrum company report. http://www. sharedspectrum.com/.

18. Federal Communications Commission, Spectrum Policy Task Force report. ET Docket (02-135), 215 (2002)

19. Q Zhao, BM Sadler, A survey of dynamic spectrum access. IEEE Signal Processing Mag. 24(3), 79-89 (2007)

20. K Patil, R Prasad, K Skouby, A survey of worldwide spectrum occupancy measurement campaigns for cognitive radio, in IEEE 2011 International Conference on Devices and Communications (ICDeCom) (Mesra, 24-25 February 2011), pp. 1-5

21. Federal Communications Commission, Second memorandum opinion and order in the matter of unlicensed operation in the TV broadcast bands (ET Docket No. 04-186) and additional spectrum for unlicensed devices 
below $900 \mathrm{MHz}$ and in the $3 \mathrm{GHz}$ band (ET Docket No. 02-380) (FCC 10-174) (Washington, D.C., September 2010)

22. J Mitola III, GQ Maguire Jr, Cognitive radio: making software radios more personal. IEEE Pers. Commun. 6(4), 13-18 (1999)

23. J Mitola III, Cognitive radio for flexible mobile multimedia communications, in IEEE International Workshop on Mobile Multimedia Communications, 1999. (MoMuC'99) (San Diego, 15-17 November 1999), pp. $3-10$

24. J Mitola III, Cognitive radio: an integrated agent architecture for software defined radio. Dissertation. PhD thesis, Royal Inst. Technol. (KTH), Stockholm, Sweden, pp. 271-350 (2000)

25. S Haykin, Cognitive radio: brain-empowered wireless communications. IEEE J. Select. Areas Commun. 23(2), 201-220 (2005)

26. IF Akyildiz, W-Y Lee, MC Vuran, S Mohanty, Next generation/dynamic spectrum access/cognitive radio wireless networks: a survey. Comput. Netw. 50(13), 2127-2159 (2006)

27. AM Wyglinski, M Nekovee, T Hou, Cognitive Radio Communications and Networks: Principles and Practice (Academic, 2009)

28. M Di Felice, R Doost-Mohammady, KR Chowdhury, L Bononi, Smart radios for smart vehicles: cognitive vehicular networks. IEEE Veh. Tech. Mag. 7(2), 26-33 (2012)

29. S Pagadarai, A Wyglinski, R Vuyyuru, Characterization of vacant UHF TV channels for vehicular dynamic spectrum access, in 2009 IEEE Vehicular Networking Conference (VNC) (Tokyo, 28-30 October 2009), pp. 1-8

30. M Di Felice, KR Chowdhury, L Bononi, Cooperative spectrum management in cognitive vehicular ad hoc networks, in 2011 IEEE Vehicular Networking Conference (VNC) (Amsterdam, November 2011), pp. 47-54, 14-16

31. H Li, DK Irick, Collaborative spectrum sensing in cognitive radio vehicular ad hoc networks: belief propagation on highway, in 2010 IEEE 71st Vehicular Technology Conference (VTC 2010-Spring) (Taipei, 16-19 May 2010), pp. 1-5

32. KTsukamoto, Y Omori, O Altintas, M Tsuru, Y Oie, On spatially-aware channel selection in dynamic spectrum access multi-hop inter-vehicle communications, in 2009 IEEE 70th Vehicular Technology Conference Fall (VTC 2009-Fall) (Anchorage, 20-23 September 2009), pp. 1-7

33. W Kim, M Gerla, SY Oh, K Lee, A Kassler, CoRoute: a new cognitive anypath vehicular routing protocol. Wireless Comm. Mobile Comput. 11(12), 1588-1602 (2011)

34. XY Wang, P-H Ho. IEEE Trans. Veh. Technol. 59(4), 1936-1948 (2010)

35. D Niyato, E Hossain, P Wang, Optimal channel access management with QoS support for cognitive vehicular networks. IEEE Trans. Mobile Comput. 10(4), 573-591 (2011)

36. IF Akyildiz, W-Y Lee, KR Chowdhury, CRAHNs: Cognitive radio ad hoc networks. Ad Hoc Networks. 7(5), 810-836 (2009)

37. ISO Technical Committee 204 WG16, Intelligent transport systems communications access for land mobiles (CALM) - architecture (2010)

38. ISO Technical Committee 204 WG16, Intelligent transport systems communications access for land mobiles (CALM) - M5, ISO 21215

39. ISO Technical Committee 204 WG16, Intelligent transport systems communication access for land mobiles (CALM) - non-IP networking Part 1: fast networking and transport layer protocol (FNTP), ISO 29281-1:2013

40. ISO Technical Committee 204 WG16, Intelligent transport systems communications access for land mobiles (CALM) - ITS station management - Part 5: fast service advertisement protocol (FSAP), ISO 24102-5:2013

41. ETSI ES 202663 v1.1.0., Intelligent Transport Systems (ITS); European profile standard for the physical and medium access control layer of Intelligent Transport Systems operating in the $5 \mathrm{GHz}$ frequency band

42. R Sevlian, C Chun, I Tan, A Bahai, K Laberteaux, Channel characterization for $700 \mathrm{MHz}$ DSRC vehicular communication. J. Electr. Comput. Eng. 2010, 40 (2010)

43. J Jakubiak, Y Koucheryavy, State of the art and research challenges for VANETs, in 5 th IEEE Consumer Communications and Networking Conference, 2008. CCNC 2008 (Las Vegas, 10-12 January 2008), pp. 912-916

44. SChen, Vehicular dynamic spectrum access: using cognitive radio for automobile networks, Ph.D. Thesis, Worcester Polytechnic Institute (2012)

45. ZD Chen, H Kung, D Vlah, Ad hoc relay wireless networks over moving vehicles on highways, in Proceedings of the 2nd ACM International
Symposium on Mobile Ad Hoc Networking \& Computing, Long Beach (ACM, New York, 2001), pp. 247-250

46. D Niculescu, B Nath, Trajectory based forwarding and its applications, in Proceedings of the 9th Annual International Conference on Mobile Computing and Networking, San Diego (ACM, New York, 2003), pp. 260-272

47. P Bose, P Morin, I Stojmenović, J Urrutia. Routing with guaranteed delivery in ad hoc wireless networks. Wireless Networks. 7(6), 609-616 (2001)

48. J-H Lee, T Ernst, IPv6 security issues in cooperative intelligent transportation systems. Comput. J. 56(10), 1189-1197 (2013)

49. J-H Lee, T Ernst, Security issues of IPv6 communications in cooperative intelligent transportation systems (poster), in 2011 IEEE Vehicular Networking Conference (VNC) (Amsterdam, 14-16 November 2011), pp. 284-290

50. J-H Lee, T Ernst, J Bonnin, Cross-layered architecture for securing IPv6 ITS communication: example of pseudonym change, in IEEE 2011 Third International Workshop on Cross Layer Design (IWCLD) (Rennes, November 2011), pp. 1-6

51. J-H Lee, T Ernst, X Ma, Performance analysis of secure beaconing messages for GeoNetworking. Secur. Comm. Netw (2011). doi: $10.1002 / \mathrm{sec} .396$

52. J Mitola, The software radio architecture. IEEE Commun. Mag. 33(5), 26-38 (1995)

53. J Mitola, Software Radio (Wiley, New York, 2000)

54. WH Tuttlebee, Software Defined Radio: Origins, Drivers, and International Perspectives (Wiley, New York, 2002)

55. M Dillinger, K Madani, N Alonistioti, Software Defined Radio: Architectures, Systems and Functions (Wiley, New York, 2005)

56. FK Jondral, Software-defined radio: basics and evolution to cognitive radio. EURASIP J. Wirel. Commun. Netw. 2005(3), 275-283 (2005)

57. H Arslan, Cognitive Radio, Software Defined Radio, and Adaptive Wireless Systems (Springer, London, 2007)

58. B Wang, KR Liu, Advances in cognitive radio networks: a survey. IEEE J. Sel. Top. Sign. Proces. 5(1), 5-23 (2011)

59. TR Newman, R Rajbanshi, AM Wyglinski, JB Evans, GJ Minden. Mobile Network Appl. 13(5), 442-451 (2008)

60. S Chen, AM Wyglinski, S Pagadarai, R Vuyyuru, O Altintas, Feasibility analysis of vehicular dynamic spectrum access via queueing theory model. IEEE Commun. Mag. 49(11), 156-163 (2011)

61. S Chen, R Vuyyuru, O Altintas, AM Wyglinski, Learning in vehicular dynamic spectrum access networks: opportunities and challenges, in IEEE International Symposium on Intelligent Signal Processing and Communications Systems (ISPACS) (Chiang Mai, 7-9 December 2011), pp. 1-6

62. S Chen, AM Wyglinski, Efficient spectrum utilization via cross-layer optimization in distributed cognitive radio networks. Comput. Comm. 32(18), 1931-1943 (2009)

63. SC Jha, MM Rashid, VK Bhargava, C Despins, Medium access control in distributed cognitive radio networks. Wireless Commun. 18(4), 41-51 (2011)

64. SC Jha, U Phuyal, MM Rashid, VK Bhargava, Design of OMC-MAC: an opportunistic multi-channel MAC with QoS provisioning for distributed cognitive radio networks. IEEE Trans. Wireless Commun. 10(10), 3414-3425 (2011)

65. S Chen, R Vuyyuru, O Altintas, AM Wyglinski, On optimizing vehicular dynamic spectrum access networks: automation and learning in mobile wireless environments, in 2011 IEEE Vehicular Networking Conference (VNC) (Amsterdam, 14-16 November 2011), pp. 39-46

66. K Hassan, R Gautier, I Dayoub, E Radoi, M Berbineau, Non-parametric multiple-antenna blind spectrum sensing by predicted eigenvalue threshold, in 2012 IEEE International Conference on Communications (ICC), (2012), pp. 1634-1629

67. S Kharbech, I Dayoub, E Simon, M Zwingelstein-Colin, Blind digital modulation detector for MIMO systems over high-speed railway channels, in Communication Technologies for Vehicles (Springer, Heidelberg, 2013), pp. 232-241

68. K Hassan, I Dayoub, W Hamouda, M Berbineau, Automatic modulation recognition using wavelet transform and neural networks in wireless systems. EURASIP J. Adv. Sig. Pr. (2010). doi:10.1155/2010/532898

69. K Hassan, I Dayoub, W Hamouda, CN Nzeza, M Berbineau, Blind digital modulation identification for spatially-correlated MIMO systems. IEEE Trans. Wireless Commun. 11(2), 683-693 (2012) 
70. E Simon, M Khalighi, Iterative Soft-Kalman channel estimation for fast time-varying MIMO-OFDM channels. IEEE Wireless Commun. Lett. 2(6), 599-602 (2013)

71. IF Akyildiz, BF Lo, R Balakrishnan, Cooperative spectrum sensing in cognitive radio networks: a survey. Phys. Commun. 4(1), 40-62 (2011)

72. AW Min, KG Shin, Impact of mobility on spectrum sensing in cognitive radio networks, in Proceedings of the 2009 ACM Workshop on Cognitive Radio Networks, Beijing, China (ACM, New York, 2009), pp. 13-18

73. K Fawaz, A Ghandour, M Olleik, H Artail, Improving reliability of safety applications in vehicle ad hoc networks through the implementation of a cognitive network, in IEEE 17th International Conference on Telecommunications (ICT) (Doha, 4-7 April 2010), pp. 798-805

74. D Borota, G Ivkovic, R Vuyyuru, O Altintas, I Seskar, P Spasojevic, On the delay to reliably detect channel availability in cooperative vehicular environments, in IEEE 73rd Vehicular Technology Conference (VTC Spring) (Yokohama, 15-18 May 2011), pp. 1-5

75. M Di Felice, KR Chowdhury, L Bononi, Analyzing the potential of cooperative cognitive radio technology on inter-vehicle communication, in 2010 IFIP Wireless Days (WD) (Venice, 20-22 October 2010), pp. 1-6

76. R Doost-Mohammady, KR Chowdhury, Design of spectrum database assisted cognitive radio vehicular networks, in 2012 7th International ICST Conference on Cognitive Radio Oriented Wireless Networks and Communications (CROWNCOM) (Stockholm, 18-20 June 2012), pp. 1-5

77. AJ Ghandour, K Fawaz, H Artail, M Di Felice, L Bononi, Improving vehicular safety message delivery through the implementation of a cognitive vehicular network. Ad Hoc Networks. 11(8), 2408-2422 (2013)

78. M Khabbaz, C Assi, A Ghrayeb, Modeling and analysis of DSA-based vehicle-to-infrastructure communication systems. IEEE Trans. Intell. Transport. Syst. 14(3), 1186-1196 (2013)

79. C Yang, Y Fu, Y Zhang, S Xie, R Yu, Energy-efficient hybrid spectrum access scheme in cognitive vehicular ad hoc networks. IEEE Commun. Lett. 17(2), 329-332 (2013)

80. KR Chowdhury, MD Felice, SEARCH: a routing protocol for mobile cognitive radio ad-hoc networks. Comput. Commun. 32(18), 1983-1997 (2009)

81. AS Cacciapuoti, M Caleffi, L Paura, Reactive routing for mobile cognitive radio ad hoc networks. Ad Hoc Networks. 10(5), 803-815 (2012)

82. R Laufer, H Dubois-Ferriere, L Kleinrock, Multirate anypath routing in wireless mesh networks, in IEEE INFOCOM 2009 (Rio de Janeiro, 19-25 April 2009), pp. 37-45

83. V Devarapalli, R Wakikawa, A Petrescu, P Thubert, Network mobility (NEMO) basic support protocol (2005). RFC 3963, January (2005)

84. J Arkko, C Perkins, D Johnson, Mobility support in IPv6 (2011). RFC 6275, July (2011)

85. S Gundavelli, K Leung, V Devarapalli, K Chowdhury, B Patil, Proxy mobile IPv6. RFC 5213, August (2008)

86. J-H Lee, T Ernst, Lightweight network mobility within PMIPv6 for transportation systems. IEEE Syst. J. 5(3), 352-361 (2011)

87. J-H Lee, T Ernst, N Chilamkurti, Performance analysis of PMIPv6-based network mobility for intelligent transportation systems. IEEE Trans. Veh. Technol. 61(1), 74-85 (2012)

88. J-H Lee, KD Singh, J-M Bonnin, S Pack, Mobile data offloading: a host-based distributed mobility management approach. IEEE Internet Comput. 18(1), 20-29 (2014)

89. L De Nardis, M-D Guirao, Mobility-aware design of cognitive radio networks: challenges and opportunities, in 2010 IEEE Proceedings of the Fifth International Conference on Cognitive Radio Oriented Wireless Networks \& Communications (CROWNCOM) (Cannes, 9-11 June 2010), pp. 1-5

90. W-Y Lee, IF Akyildiz, Spectrum-aware mobility management in cognitive radio cellular networks. IEEE Trans. Mobile Comput. 11(4), 529-542 (2012)

91. T Wang, L Song, Z Han, Coalitional graph games for popular content distribution in cognitive radio VANETs. IEEE Trans. Veh. Technol. 62(8) 4010-4019 (2013). http://dx.doi.org/10.1109/TVT.2013.2245353

92. CORRIDOR: Cognitive radio for railway through dynamic and opportunistic spectrum reuse. http://corridor.ifsttar.fr. Accessed on 11 March 2014

93. N Haziza, M Kassab, R Knopp, J Harri, F Kaltenberger, P Agostini, M Berbineau, C Gransart, J Besnier, J Ehrlich, H Aniss, Multi-technology vehicular cooperative system based on software defined radio (SDR), in
NET4CARS 2013, 5th International Workshop on Communication Technologies for Vehicles, Lille, May 14-15, 2013 (Springer, Heidelberg, 2013), pp. 84-95

94. A Amanna, M Gadhiok, MJ Price, JH Reed, W Siriwongpairat, THimsoon, Railway cognitive radio. IEEE Veh. Tech. Mag. 5(3), 82-89 (2010)

95. CORNET, Cognitive radio network testbed. http://cornet.wireless.vt.edu. Accessed on 11 March 2014.

96. M Gadhiok, A Amanna, MJ Price, JH Reed, Metacognition: enhancing the performance of a cognitive radio, in 2011 IEEE First International Multi-Disciplinary Conference on Cognitive Methods in Situation Awareness and Decision Support (CogSIMA) (Miami, Beach, 22-24 February 2011), pp. 198-203

97. A Amoroso, G Marfia, M Roccetti, G Pau, Creative testbeds for VANET research: a new methodology, in 2012 IEEE Consumer Communications and Networking Conference (CCNC) (Las Vegas, 14-17 January 2012), pp. 477-481

98. G Marfia, M Roccetti, A Amoroso, M Gerla, G Pau, J-H Lim, Cognitive cars: constructing a cognitive playground for VANET research testbeds, in Proceedings of the 4th International Conference on Cognitive Radio and Advanced Spectrum Management Barcelona, 26-29 October 2011 (ACM, New York, 2011), pp. 29:1-29:5

99. Open-Access Research Testbed for Next-Generation Wireless Networks (ORBIT). WINLAB, Rutgers University. http://www.orbit-lab.org. Accessed on 11 March 2014

100. KR Chowdhury, M Di Felice, IF Akyildiz, TP-CRAHN: a transport protocol for cognitive radio ad-hoc networks, in IEEE INFOCOM 2009 (Rio de Janeiro, 19-25 April 2009), pp. 2482-2490

101. KD Singh, J-M Bonnin, D Ros, Optimising TCP over cognitive radio networks for trains, in IEEE 2012 12th International Conference on ITS Telecommunications (ITST) (Taipei, 5-8 November 2012), pp. 678-682

102. G Zhang, Y Xu, X Wang, X Tian, J Liu, X Gan, H Yu, L Qian, Multicast capacity for VANETs with directional antenna and delay constraint. IEEE J. Select. Areas Commun. 30(4), 818-833 (2012)

103. KD Singh, A Ksentini, B Marienval, Quality of experience measurement tool for SVC video coding, in 2011 IEEE International Conference on Communications (ICC) (Kyoto, 5-9 June 2011), pp. 1-5

104. KD Singh, Y Hadjadj-Aoul, G Rubino, Quality of experience estimation for adaptive HTTP/TCP video streaming using H. 264/AVC, in 2012 IEEE Consumer Communications and Networking Conference (CCNC) (Las Vegas, 14-17 January 2012), pp. 127-131

105. K Deep Singh, K Piamrat, H Park, C Viho, J-M Bonnin, Optimising QoE for scalable video multicast over WLAN, in 2013 IEEE 24th International Symposium on Personal Indoor and Mobile Radio Communications (PIMRC) (London, 8-11 September 2013), pp. 2131-2136

106. O Altintas, M Nishibori, T Oshida, C Yoshimura, Y Fujii, K Nishida, Y Ihara, M Saito, K Tsukamoto, M Tsuru, Y Oie, R Vuyyuru, A Al Abbasi, M Ohtake, M Ohta, T Fujii, Si Chen, S Pagadarai, M Alexander Wyglinski, Demonstration of vehicle to vehicle communications over TV white space (Demo paper), in 2011 IEEE Vehicular Technology Conference (VTC Fall) (San Francisco, 5-8 September 2011), pp. 1-3

107. T Rzayev, Y Shi, A Vafeiadis, S Pagadarai, AM Wyglinski, Implementation of a vehicular networking architecture supporting dynamic spectrum access, in 2011 IEEE Vehicular Networking Conference (VNC) (Amsterdam, 14-16 November 2011), pp. 33-38

108. SUMO, Simulation of urban mobility. http://sumo-sim.org. Accessed on 11 March 2014

\section{doi:10.1186/1687-1499-2014-49}

Cite this article as: Singh et al: Cognitive radio for vehicular ad hoc networks (CR-VANETs): approaches and challenges. EURASIP Journal on Wireless Communications and Networking 2014 2014:49. 